\title{
Current circulation and equilibrium in toroidal electron cyclotron resonance (ECR) plasmas in the LATE device
}

\section{AUTHOR(S):}

Nishi, S; Sakabe, T; Uchida, M; Tanaka, H; Maekawa, T

\section{CITATION:}

Nishi, S ...[et al]. Current circulation and equilibrium in toroidal electron cyclotron resonance (ECR) plasmas in the LATE device. Plasma Physics and Controlled Fusion 2010, 52(12): 125004.

\section{ISSUE DATE:}

2010-11-03

URL:

http://hdl.handle.net/2433/197315

\section{RIGHT:}

This is an author-created, un-copyedited version of an article accepted for publication in Plasma Physics and Controlled Fusion. The publisher is not responsible for any errors or omissions in this version of the manuscript or any version derived from it. The Version of Record is available online at http://dx.doi.org/10.1088/0741-3335/52/12/125004; This is not the published version. Please cite only the published version.; この論文は出版社版でありません。引用の際 には出版社版をご確認ご利用ください。 


\title{
Current circulation and equilibrium in toroidal ECR plasmas in the LATE device
}

\author{
S Nishi, T Sakabe, M Uchida, H Tanaka and T Maekawa \\ Graduate School of Energy Science, Kyoto University, Kyoto 606-8502, Japan \\ E-mail: maekawa@energy.kyoto-u.ac.jp
}

\begin{abstract}
In toroidal plasmas immersed in a toroidal field of $B_{\phi}$, the electrons drift downwards, while the ions drift upwards due to the field gradient and curvature. The electrons that drift down to the bottom would return through the conducting vessel to the top, on which they recombine with the ions, completing the current circulation. Helical field lines by the superposition of a vertical field $B_{Z}$ would provide another return path of internal current circulation, along which the electrons flow toroidally, generating a toroidal current. These conjectures have been examined on ECR plasmas in the Low Aspect ratio Torus Experiment device with current-collecting electrodes at the top and bottom of the vacuum chamber. Upon the blocking of external return path the discharges terminate when $B_{Z}=0$, while they survive with a toroidal current when $B_{Z}$ is applied, showing that current circulation is vital to maintain the discharge. The detailed studies reveal the characteristics of current circulation and equilibrium. When $B_{Z}=0$, the electron pressure profile is a uniform vertical ridge along the ECR layer in accordance with the electron vertical drift current circulating via the external circuit, while an upwardly-shifted potential hill arises, providing the ions $\mathbf{E} \times \mathbf{B}$ drift paths around the potential peak to the vicinity of the top electrode. When a $B_{Z}$ is applied, the central electron pressure rises with an upwardly shifted peak, being relaxed from the vertical uniformity by addition of the internal return circuit. The space potential $V_{S}$ decreases and the electron density obeys the Boltzmann law under $V_{S}$. The vertical and toroidal currents are found to be equilibrium currents driven by the base and excess portions of electron pressure, respectively, to ensure the radial force balance of the plasma torus.
\end{abstract}




\section{Introduction}

There is considerable interest in electron cyclotron resonance (ECR) heated plasmas produced in a purely toroidal magnetic field or in a helical field by superposition of a weak vertical field $B_{Z}$ but with no rotational transform. By injection of microwaves at the ECR frequency, breakdown easily takes place at the ECR layer, after which the discharge is maintained by the deposition of the microwave power at the ECR layer and/or the upper hybrid resonance (UHR) layer [1,2]. This is a standard method of pre-ionization and heating to assist the start-up of plasma current in tokamaks [3]. This technique is intended to be used for the International Thermonuclear Experimental Reactor tokamak [4]. They also served as a target plasma for solenoid-free startup of tokamaks by lower hybrid waves $[5,6]$ and their characteristics were investigated in connection with confinement and equilibrium in the open field configuration $[7,8]$. In addition to the usage in the large scale experiments for magnetic fusion such as ITER with the frequencies over $100 \mathrm{GHz}$ and the power level over $1 \mathrm{MW}$, ECR-heated plasmas in small toroidal devices, which are often produced by $2.45 \mathrm{GHz}$ microwave power at the power level of $1 \mathrm{~kW}$, have been frequently used as a useful bed for basic studies in plasma physics. In the TORPEX device various phenomena including particle confinement [9], charged particle production [10] and blob generation [11] were investigated. In the Helimak device drift wave instability was studied [12]. In the Blaaman device particle transport by the turbulence was studied [13]. In the CDX device [14, 15] and also in the LATE device $[16,17]$ it was found that a toroidal current was generated when $B_{Z}$ was superposed.

In axisymmetric toroidal plasmas immersed in the toroidal magnetic field $B_{\phi}$ in the cylindrical coordinates $(R, \phi, Z)$ system with Z-axis being the symmetric axis, each charged particle drifts vertically across the field lines due to the radial gradient and curvature of $B_{\phi}$ with the velocity given by

$$
V_{V T F}=\frac{m\left(v_{\|}^{2}+v_{\perp}^{2} / 2\right)}{q B_{\phi} R}
$$

Here, $B_{\phi}$ is the toroidal field at the radial coordinate $R ; q$ and $m$ are the charge and mass ; and $\|$ and $\perp$ denote the parallel and perpendicular components of the velocity to the field, respectively. Hereafter, this vertical drift is referred to as vacuum-toroidalfield (VTF) drift since the torodal field in these plasmas is essentially vacuum field with negligibly small paramagnetic and diamagnetic effects. The local current density by electron (ion) VTF-drift is given by summing up the VTF-drift current of every electron (ion) over the Maxwell velocity distribution and is written in terms of the local pressure $p_{e}\left(p_{i}\right)$;

$$
j_{V T F e(i)}=\frac{2 p_{e(i)}}{R B_{\phi}}
$$

Equation (1) predicts that the electrons drift downwards while the positive ions drift upwards (we consider the case of $B_{\phi}>0$ without loss of generality.), generating a vertical charge separation current given by equation (2). The equilibrium characteristics of this 
type of plasmas may be primarily governed by the circulation of the charge separation current as predicted theoretically $[8,18]$. While experimental verification on them is of basic importance, few experiments on only fragmentary aspects were reported [18, 19]. Here we study, in a comprehensive manner, various aspects and characteristics of the current circulation and equilibrium observed in ECR plasmas in the LATE device.

When $B_{Z}=0$, it might seen that this vertical charge separation current accumulates enormous amount of charge, positive charge at the top and negative one at the bottom of the plasma since the plasma is limited at both the upper and lower sides by the vacuum vessel. Such a large accumulation would build up a strong vertical electric field $E_{z}$ and the plasma would be swept out by the fast $E_{z} \times B_{\phi}$ drift. However, experiments show that ECR discharges are maintained as far as the microwave power is fed to the plasma. This result suggests that actually, the charge separation current circulates via some return path, leaving only a very low level of charge accumulation in the plasma.

Usually the plasmas are surrounded by the conducting vessel that would provide a return path for the vertical charge separation current. In order to complete the current circulation equal amount of current must flow through the two plasma-wall interfaces at the top and bottom of the chamber. It is conjectured that the VTF-drift electrons reach the bottom, flow into the conducting vessel, go up through the vessel to the top, on which surface they recombine with the ions that flow up from the plasma. Any imbalance of these two currents of electrons and ions causes charge accumulation in the plasma. Experimental observations deny infinite charge accumulation in steady toroidal ECR discharges, indicating that a balance is always reached between the two currents carried by different signs of charges.

This looks, at the first glance, impossible as far as we take only the VTF-drifts of electrons and ions into account since the electron temperature is much higher than the ion temperature in ECR plasmas and the electron current and ion current predicted by equation (2) are much different. Net charge would accumulate in the plasma unless electrons emitted freely into the plasma from the ion-side wall. Note that while electrons flow freely into the conducting wall, the reverse is prevented since they must overcome the potential gap (work function) to get through the wall surface out into the plasma. Thus, there arise two questions. How does the circulating current relate with the electron pressure profile, and what makes the two currents of electrons and ions balanced.

The circulation of the charge separation current via the conducting liner was first invoked by S. Yoshikawa et al. in 1963 [18] and recently confirmed by using a pair of current collecting electrodes fabricated at the top and bottom of the discharge chamber in the LATE device [19]. The paper [19], however, only gave a limited answer to the first question. A comprehensive answer will be given here. Furthermore, the second question totally remained and will be addressed in the present paper.

When a weak $B_{Z}$ field is superposed to the toroidal field, the field line becomes helical that provides another return path for the charge separation current. The electrons can flow back along the helical field lines to compensate the charge separation. This return current has both the toroidal and vertical components. The downward 
vertical component exactly cancels out the corresponding upward VTF drift current of electrons and, as a result, a purely toroidal current remains. Thus, some portion of the vertical charge separation current of electrons would be converted to a toroidal current and the remainder circulates via the external circuit.

At this point it is useful to consider the problem in terms of the radial force balance of plasma loop [8]. Toroidal plasmas have finite pressure and they tend to expand horizontally by pressure ballooning effect. Therefore, there must be a counter force to balance this expanding force. In the plasmas immersed in the simple toroidal field such counter force is generated only when there is a vertical current flowing in the plasma. The inward force generated by the interaction with the toroidal field can counter balance the ballooning force. In the case with a weak $B_{Z}$ field some portion of the vertical charge separation current would be converted to the toroidal current as described above. Another inward force is generated from the toroidal current by the interaction with the $B_{Z}$ field. Thus both the vertical charge separation current and the toroidal current as appeared as the return current along the helical field lines are a sort of equilibrium current. Both currents originate from the electron VTF drift, which is driven by the thermal energy of electrons provided by the ECR heating.

While some toroidal plasma current was indeed observed when a weak vertical magnetic field $B_{Z}$ was superposed to the toroidal field [14, 15, 17], it has been unknown what portion of the vertical charge separation current in the plasma cross section is converted to the toroidal current and what portion remains and circulates via the external circuit. How does this change as $B_{Z}$ is increased?

Thus, there are a number of questions. In order to address them we have performed three series of experiments.

In the first series of experiments, a resistance is inserted into the external circuit to block the current circulation during discharges. The discharges under no $B_{z}$ field terminate instantly upon the current blocking. The discharges under sufficient magnitude of $B_{Z}$ have a significant toroidal current in addition to the external circulation current before the current blocking. Upon the current blocking these discharges survive with an increase of the toroidal current. Thus the experiments show most clearly that current circulation is vital to maintain the discharge.

The second series constitutes the main part of experiments, in which we measure the two dimensional profiles of the electron temperature, density and space potential in the space between the top and bottom electrodes by changing the location of the Langmuir probe by shot by shot basis. We analyze the results based on the fluids theory and also on the particle drift orbit theory. While the guiding-centre-drift description is intuitive and, therefore, quite effective to guide us to understand the basic physics, it is not always straightforward to relate the description with the measurable quantities such as the electron density and pressure, space potential and various currents detected by the current collecting electrodes and the flux loops. The fluid description directly handles these quantities, and therefore, is useful for quantitative analyses, although physical meaning of its results is sometimes subtle. The question that how do the ions arrive at 
the top electrode is considered by the particle orbit theory. We use both descriptions to reach better understanding of the experimental results.

The third series of experiments is the same as the second series except for the working gasses. In the first and second series hydrogen gas was used. In the third series we used helium, neon and argon to investigate the roles of ions.

In the section 2 we describe the experimental apparatus. In the section 3 the three series of experiments are presented with brief analyses and discussions. Full analyses and discussions are given in section 4 . The results are summarized in section 5 .

\section{Experimental apparatus}

Figure 1 shows the LATE device. The vacuum vessel is a cylinder with an inner diameter of $100 \mathrm{~cm}$ and a height of $100 \mathrm{~cm}$. The centre post is also a cylinder with an outside diameter of $11.4 \mathrm{~cm}$, enclosing 60 turns of copper conductors for generation of the toroidal field. Both the vessel and the centre post are made of stainless steel and connected without electrical insulation. There are four sets of poloidal field coils to generate vertical magnetic fields.

By taking advantage of the low-aspect-ratio cylindrical space of rectangular cross section, we have installed top and bottom electrodes to collect the vertical charge separation current. Each electrode is composed of a panel and a limiter. The panel consists of twelve trapezoidal plates attached to a common frame. Because of the lowaspect-ratio feature, the panels have a large area and almost cover the floor and the ceiling of the vacuum vessel. Therefore, the interaction between the side walls of the vacuum vessel and the plasma is small. The limiter is a rectangular board attached to the panel at one toroidal section. The panels and limiters are made of stainless steel.

Each of the top and bottom panels, the top and bottom limiters, and the vacuum vessel is independently connected to the common ground point through a current pickup resistor $(0.1 \Omega)$. Thus all the currents flowing from the plasma into the electrodes and the vessel can be monitored. Observed currents are less than $5 \mathrm{~A}$. Therefore, potential drops through the pickup resistors are less than $0.5 \mathrm{~V}$, which are negligible compared with the potential difference between the plasma core and the vessel.

The interlock switches are inserted in the external circuit for the insertion of current blocking resistances. They are normally closed, making a closed circuit for circulation of the vertical charge-separation current. When they are opened, the current circulation is strongly impeded since the current must flow through the resistance of $80 \Omega$ in the external circuit.

A Langmuir probe is rotated by a motor around its vertical axis and is also slid vertically by another motor Thus the probe tip covers the whole range from $R=10 \mathrm{~cm}$ to $45 \mathrm{~cm}$ and also from $Z=-24 \mathrm{~cm}$ to $+24 \mathrm{~cm}$ for the measurement of the profiles of the electron density and temperature and the space potential on the poloidal cross section. Furthermore, the line-integrated electron density along a chord on the mid-plane that has a tangential radius of $12 \mathrm{~cm}$ is measured by a $70 \mathrm{GHz}$ interferometer 
(a) Top view

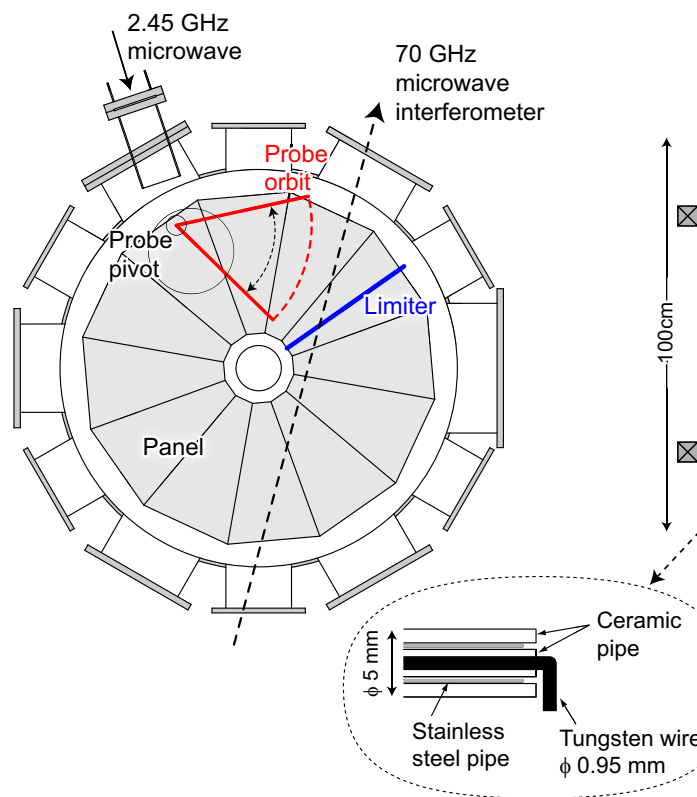

(b) Side view

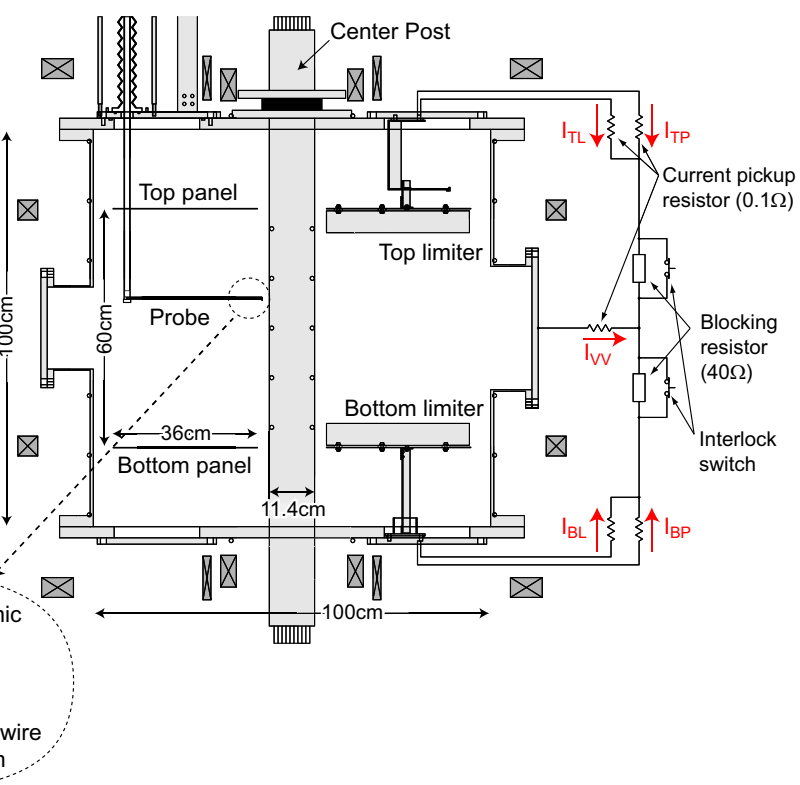

Figure 1. Top and side views of the LATE device with an enlargement of the probe tip

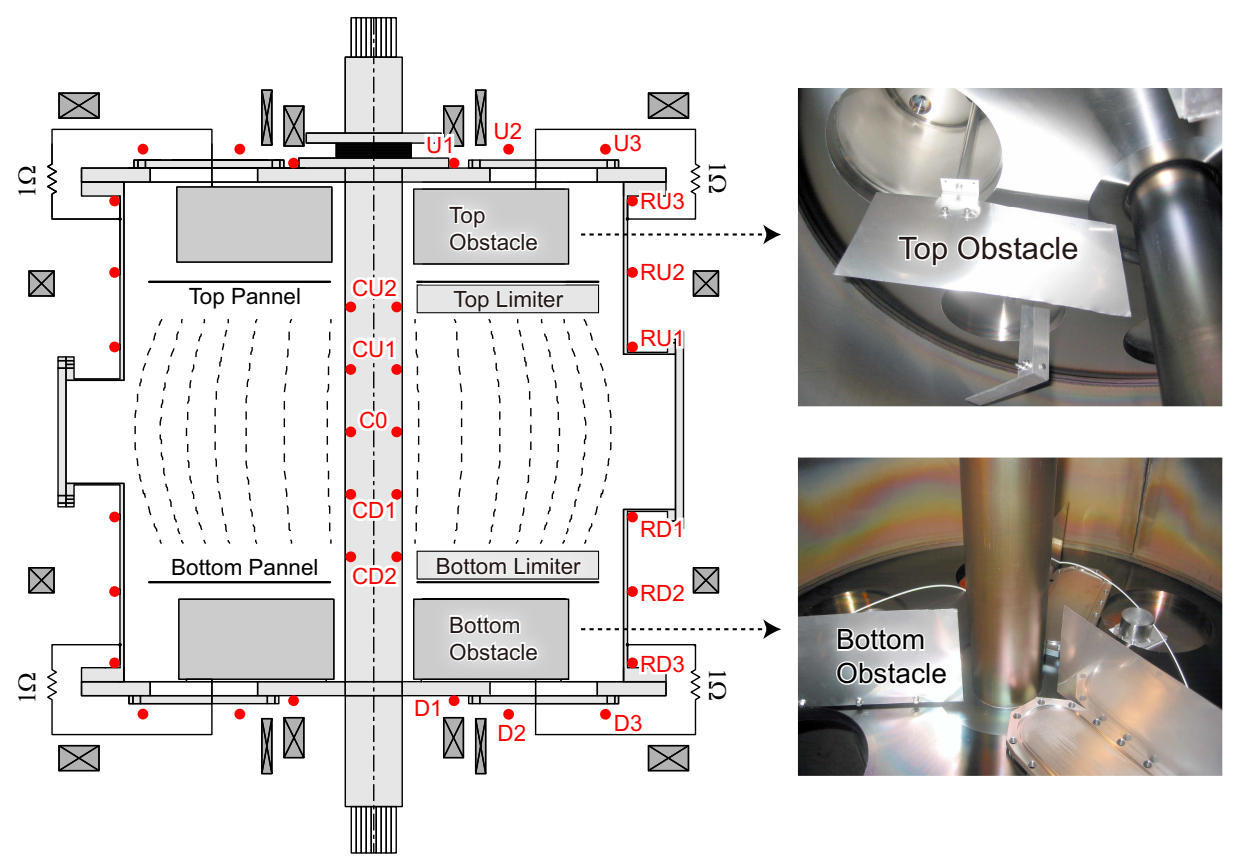

Figure 2. Field-line-cutting obstacle plates for suppression of discharge behind the top and bottom panels, locations of seventeen flux loops (C0, CU1, etc.) and field lines of external vertical field. 
Microwave power at $2.45 \mathrm{GHz}$ generated by a magnetron $\left(P_{i n j} \leq 5 \mathrm{~kW}, \tau \leq 2 \mathrm{~s}\right)$ is injected by a launcher of open circular waveguide at a radial port. The angle between the axis of the launcher and the toroidal field is $\sim 80$ degrees at the injection point of $R=50 \mathrm{~cm}$ and the wave is linearly polarized with the electric field parallel to the horizontal plane, indicating that almost power is injected in the $\mathrm{O}$ mode.

Information on the toroidal plasma current and its profile is obtained by using the flux signals from the seventeen flux loops shown in figure 2. Thus, the various plasma profiles by the movable Langmuir probe, the currents flowing into the top and bottom electrodes and the flux signals from the toroidal plasma current constitute the basic data set of information to address the various questions raised in the introduction.

An ECR discharge sometimes takes place at the spaces behind the top and bottom panels. Then, some currents flow into the panel from behind. We can not isolate the current from the main chamber from these currents. Thus the discharges behind the panels disturb the experiments. To avoid the discharge in these spaces several plates are fabricated to intersect the filed lines as shown in figure 2. These obstacle plates have also current pick-up resistances by which we monitor the currents into the plates to see whether or not discharges take place behind the panels.

\section{Experimental results}

\subsection{Discharges with and without Bz and blocking of external return current}

Let us begin with a discharge under purely toroidal field shown in figure 3(a). First, toroidal coil current is ramped-up and then kept constant by a transistor power supply to produce the EC resonance layer for $2.45 \mathrm{GHz}$ at $R_{E C R}=13.7 \mathrm{~cm}\left(B_{\phi}=480 \mathrm{G}\right.$ at $R=25 \mathrm{~cm}$ ), which is the same through out the experiments. Second, puffs of hydrogen gas are introduced into the chamber to fill up the gas around the pressure of $p_{H_{2}}=3 \times 10^{-2} \mathrm{~Pa}$. Third, a microwave pulse is injected at the power $P_{i n j}=1.5 \mathrm{~kW}$. Then the breakdown takes place immediately and a toroidal plasma appears quickly as seen on the signals of the plasma light and the line electron density. The hydrogen gas pressure gradually decreases and reaches $\sim 6 \times 10^{-3}$ Pa during the initial transition time of $\sim 0.4 \mathrm{~s}$. Then, the pressure is kept constant by additional weak gas puffing.

No toroidal current is detected throughout the discharge in the present case of $B_{Z}=0$.

The currents flowing into the panels, limiters and vacuum vessel are shown in the figure. $I_{T P}$ represents the top panel current that flows into the top panel. In the same way, $I_{T L}$ represents the top limiter current, $I_{B L}$ the bottom limiter current, $I_{B P}$ the bottom panel current and $I_{V V}$ the vacuum vessel current, respectively. All the currents become steady after initial transition time. The sign is defined positive for the current direction of flowing into the conductor from the plasma throughout the paper. Here, the toroidal field direction is counter-clockwise viewing from the top $\left(B_{\phi}>0\right)$ and the electron VTF drift direction is downward. 
The bottom limiter current $I_{B L}$ is negative $(-3.6 \mathrm{~A})$, suggesting that this current is due to the electrons that VTF-drift downward. Once the electrons reach the shadow of the bottom limiter they would quickly flow into the limiter by travelling along the field lines at the thermal speed. Current into the bottom panel is hardly observed $\left(I_{B P} \simeq-0.05 \mathrm{~A}\right)$. This is consistent with the above picture of electron behavior. On the other hand, the top panel current $I_{T P}$ is positive $(+3.2 \mathrm{~A})$, showing that this current is due to ions that drift upwards and reach the top panel. The top limiter current is extremely small $\left(I_{T L} \approx-0.1 \mathrm{~A}\right)$. Thus, the combination of the limiter and the panel elucidates the characters of current carriers of the vertical charge separation currents to the top and bottom electrodes, respectively.

The vessel current $\left(I_{V V} \approx 0.5 \mathrm{~A}\right)$ is much smaller than $I_{T P}$ in the present microwave power level of $\sim 1.5 \mathrm{~kW}$. Thus, the relationship $I_{B L}+I_{T P}=0$ approximately holds. The relationship $I_{B P}+I_{B L}+I_{T L}+I_{T P}+I_{V V}=0$ is indeed always held within the accuracy of current measurement of $\sim 0.01 \mathrm{~A}$. Above results indicate that the current flowing into the top electrode is returned to the bottom electrode through the external circuit, completing current circulation of the vertical charge-separation current.

When the blocking resistance of $80 \Omega$ is inserted in the external circuit at $t=1.015$ $\mathrm{s}$, the plasma light, the electron density and all the electrode currents immediately disappear, that is, the discharge terminates. Note that the interlock system watches the plasma light signal and shuts off the microwave power about $0.04 \mathrm{~s}$ after the termination of the light signal. The decay time of the plasma image taken by a fast CCD camera is as short as $\sim 0.1 \mathrm{~ms}$ as shown in figure $4(\mathrm{~b})$. The detail of the termination is reported in the previous paper [19].

Figure $3(\mathrm{~b})$ shows a discharge with $B_{Z}=4.4 \mathrm{G}$. This is the value at $R=25 \mathrm{~cm}$ and $Z=0 \mathrm{~cm}$. Overall $B_{Z}$ field is shown in figure 2. By following the same way as the previous discharge under no $B_{Z}$ the present discharge reaches a steady phase about $0.4 \mathrm{~s}$ after the breakdown. Most remarkable change from the $B_{Z}=0$ case is the appearance of a toroidal current. Another change is that a negative current flows into the top limiter. This may have to do with that the limiter is directly connected via helical field lines with the plasma in the main discharge room between the top and bottom limiters under the finite $B_{Z}$. The bottom panel current is almost zero and the vacuum vessel current $(0.3 \mathrm{~A})$ is small except for the initial transition time. These are the same as the $B_{Z}=0$ case. In the steady phase after $t=0.35 \mathrm{~s}, I_{T P}(4.5 A)+I_{T L}(-1.3 A)=3.2 \mathrm{~A}$ while $I_{B L}+I_{B P}=I_{B L}=-3.5 \mathrm{~A}$. Thus the net current flowing into the top electrode and the net current flowing out from the bottom electrode are almost the same, indicating that the external current circulation also takes place as well in the present case.

When the blocking resistance of $80 \Omega$ is inserted in the external circuit at $t=1.01794 \mathrm{~s}$, the discharge survives and continues until the end of the microwave pulse. Both $I_{T P}$ and $I_{T L}$ change quickly upon the blocking to second steady values of $I_{T P}=2.5 \mathrm{~A}$ and $I_{T L}=-2.5 \mathrm{~A}$, respectively. Also, both $I_{B P}$ and $I_{B L}$ change quickly to their second steady values of $I_{B P}=1.0 \mathrm{~A}$ and $I_{B L}=-1.1 \mathrm{~A}$, respectively. Thus, both the total current flowing to the top electrode and the total current flowing out from the 


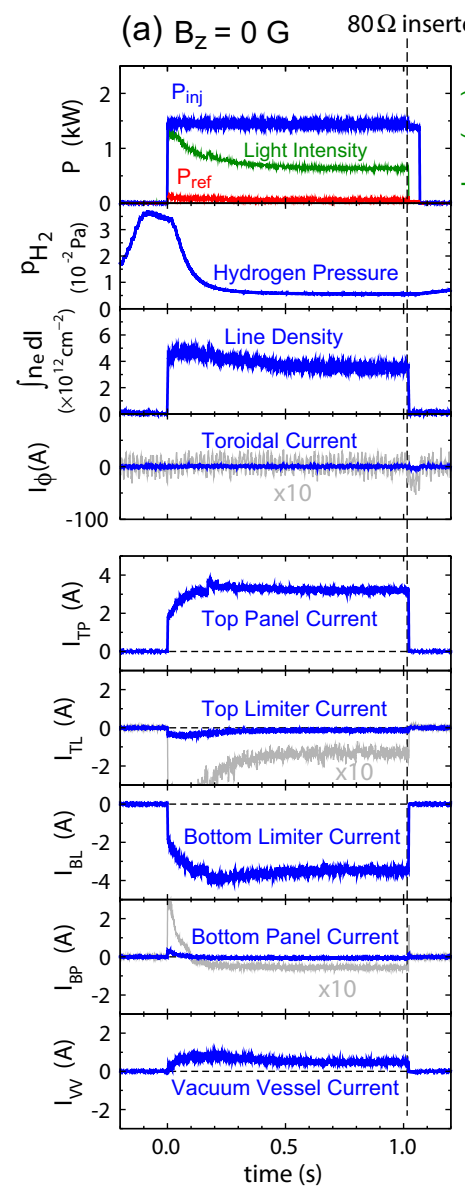

(b) $B_{z}=4.4 G \quad 80 \Omega$ inserted
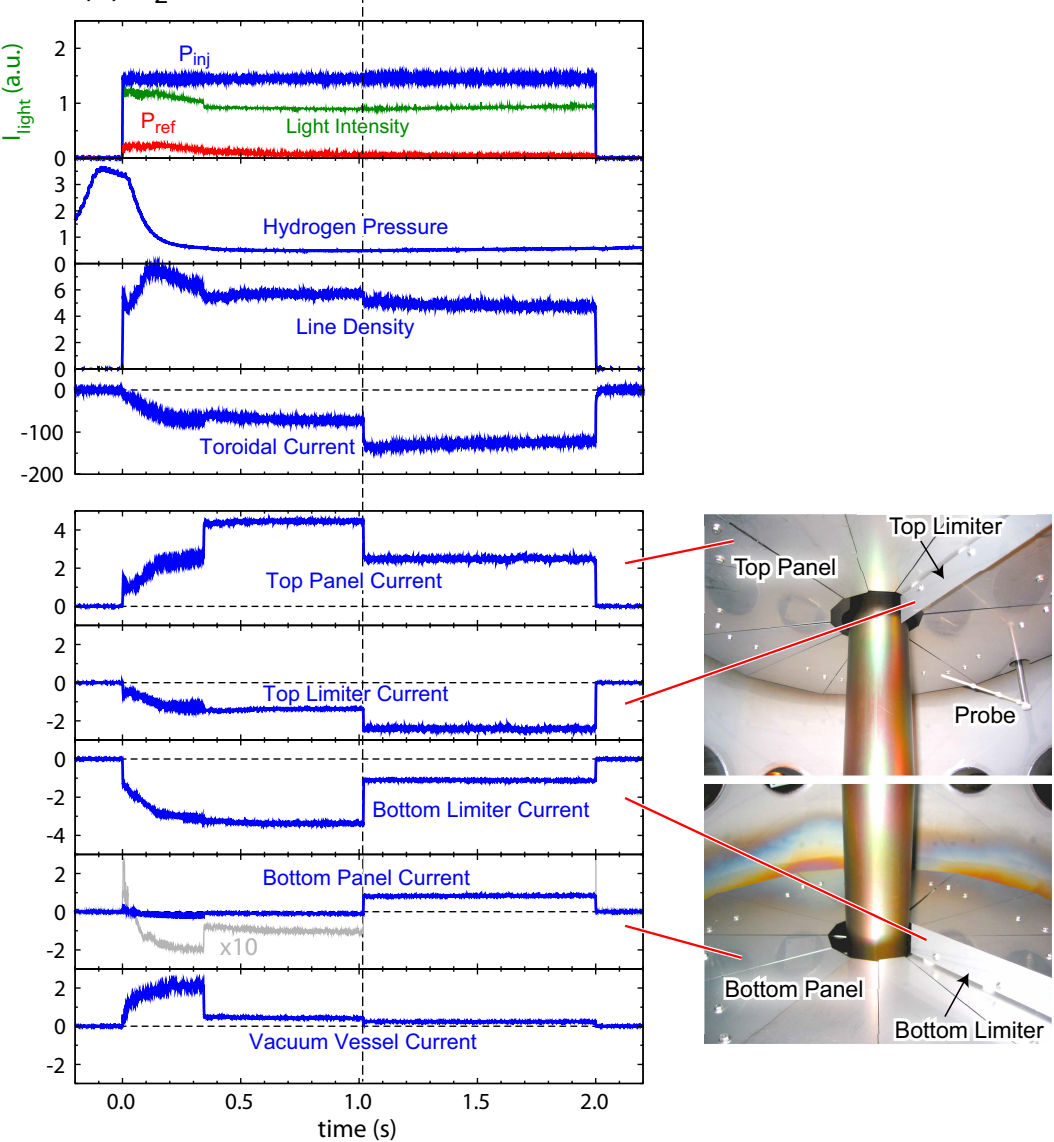

Figure 3. Typical discharges with $B_{Z}=0 \mathrm{G}$ (a) and $4.4 \mathrm{G}$ (b). The sign of electrode currents is positive when it flows into the conductor.

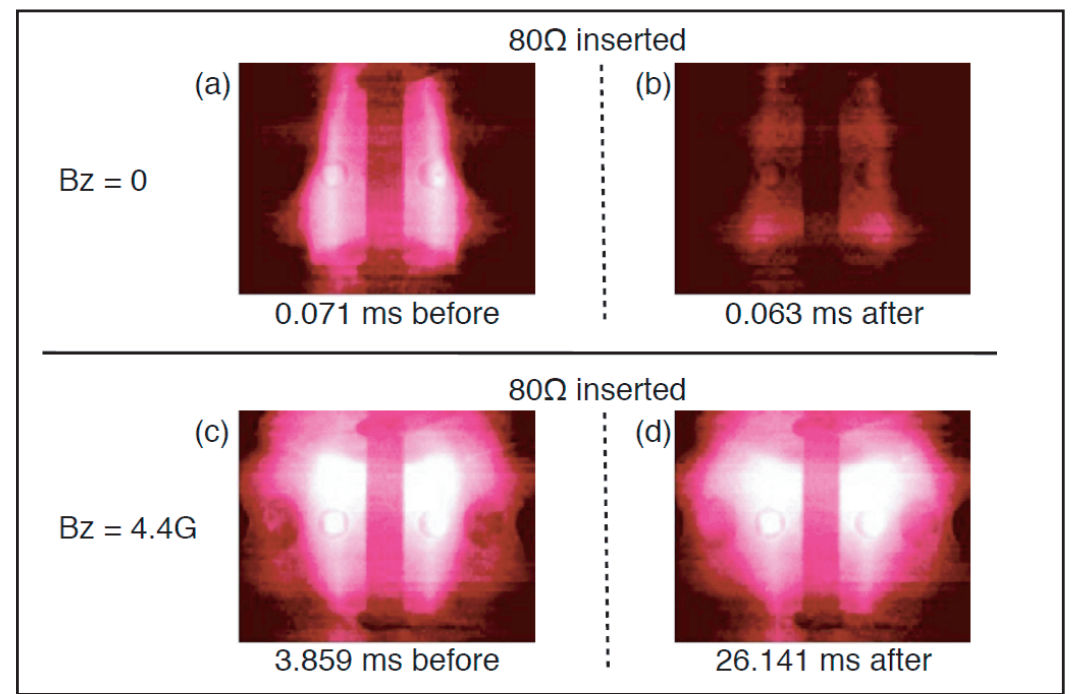

Figure 4. CCD camera images of plasmas before and after the external current blocking in the discharges under $B_{Z}=0((\mathrm{a})$ and (b)) and $4.4 \mathrm{G}((\mathrm{c})$ and (d)) in figure 3 , respectively. 
bottom electrode are almost zero after the blocking, indicating that the external current circulation terminates. Upon the blocking, on the other hand, the toroidal current $I_{\phi}$ increases almost twice.

Figures 4(c) and (d) show CCD camera images of the plasma before and after the external current blocking. The image expands after the blocking. It is also noted that the image with $B_{Z}$ (figure 4(c)) shifts upward (the proton VTF drift side) with an expansion to the lower field side compared with the image with no $B_{Z}$ (figure $4(\mathrm{a})$ ).

Discharge characteristics upon the external current blocking under various $B_{Z}$ from 0 to $5.3 \mathrm{G}$ are summarized in figure 5 , where the line integrated density shows whether the discharge is maintained or not, the sum $I_{T P}+I_{T L}$ represents the external return current and the toroidal current $I_{\phi}$ does the internal return current. In all cases the external return current terminates upon the insertion of $80 \Omega$. Behavior of internal current is remarkable. When $B_{Z}>3.5 \mathrm{G}, I_{\phi}$ increases upon the blocking, indicating that the external return current changes its return path to the internal channel upon the closing of the external return path. When $B_{Z}=2.6 \mathrm{G}$, the discharge survives although the line density decreases significantly and $I_{\phi}$ decreases slightly upon the blocking. When $B_{Z} \leq 1.7 \mathrm{G}$, the discharges terminates even if $I_{\phi}$ is finite before the blocking.

With the superposition of a weak $B_{Z}$ field to the toroidal field, the electrons can flow back along the helical field line to compensate the charge separation. Thus, a part of the vertical charge separation current would circulate via the internal helical path and the remainder would circulate via the external circuit.

The vertical charge separation current is proportional to the local electron pressure as shown by equation (2), where we neglect ion contribution since the ion temperature is much lower than the electron temperature in ECR plasmas [20]. We may divide the electron pressure into two parts for a phenomenological analysis; $p_{0}$ corresponding to the external circulation and the rest, $p_{e}-p_{0}$, to the internal circulation. Then the local density of the vertical charge separation current that returns internally is given by $j_{V T F e}($ rtrn intrnlly $)=2\left(p_{e}-p_{0}\right) / R B_{\phi}$ The internal return current has the following form in vector representation; $\mathbf{j}_{r t r n}=j_{r t r n \phi} \widehat{\phi}+j_{r t r n Z} \widehat{Z}$. Note that $j_{r t r n Z} / j_{r t r n \phi}=B_{Z} / B_{\phi}$, and $j_{r t r n Z}=-j_{V T F e}($ rtrn intrnlly $)=-2\left(p_{e}-p_{0}\right) / R B_{\phi}$. Thus,

$$
j_{r t r n \phi}=j_{r t r n Z} \frac{B_{\phi}}{B_{z}}=-\frac{2\left(p_{e}-p_{0}\right)}{R B_{z}}
$$

It is also noted that,

$$
j_{r \operatorname{rtn} \phi} \widehat{\phi} \times B_{Z} \widehat{Z}=-\frac{2\left(p_{e}-p_{0}\right)}{R} \widehat{R}
$$

In the case with no internal return current the inward $j_{V T F e} \widehat{Z} \times B_{\phi} \widehat{\phi}$ force (actually force density in the unit of $\mathrm{Nm}^{-3}$ ) counterbalances the outward pressure ballooning force $2 p_{e} / R$ as shown by equation (2). In the case that all the charge separation current returns internally, there is no vertical component of current and the inward $j_{r t r n \phi} \widehat{\phi} \times B_{Z} \widehat{Z}$ force counterbalances the ballooning force as shown by equation (4) with $p_{0}=0$. In general cases a part of vertical charge separation current still circulates via the external circuit and the remainder returns along the helical field lines. A measure for the ratio 

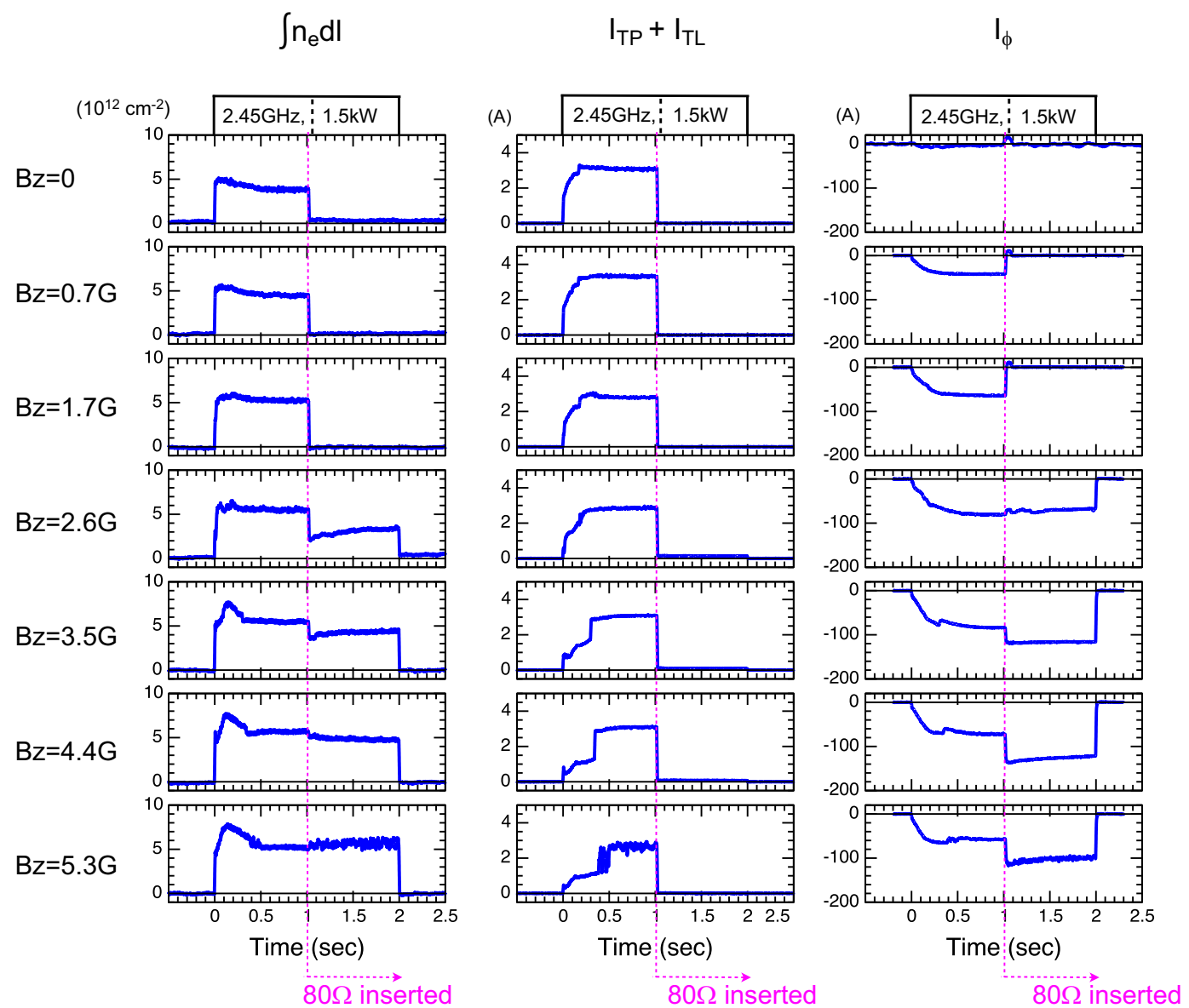

Figure 5. Discharge evolutions under various vertical fields $B_{Z}$ with a blocking of external current circuit. Line integrated electron density, external circulation current $\left(I_{T P}+I_{T L}\right)$ and internal circulation current $\left(I_{\phi}\right)$ are plotted as functions of time.

of the contribution of the vertical current to the toroidal current in counterbalancing the outward ballooning force is given by

$$
\frac{-j_{\text {rtrn } \phi} B_{Z}}{\left[j_{V T F e}-j_{V T F e}(\text { rtrn intrnlly })\right] B_{\phi}} \sim \frac{\frac{-I_{\phi}}{h w} B_{Z}}{\frac{I_{T P}+I_{T L}}{2 \pi R w} B_{\phi}}=\frac{2 \pi R}{h} \frac{-I_{\phi}}{I_{T P}+I_{T L}} \frac{B_{Z}}{B_{\phi}}
$$

Here, $w$ and $h$ are the width and height of the plasma cross section, respectively. In the discharge in figure 3(b), $I_{\phi} \sim-80 \mathrm{~A}, I_{T P}+I_{T L} \sim 3 \mathrm{~A}, R=25 \mathrm{~cm}, h=48 \mathrm{~cm}, B_{Z}=4.4$ $\mathrm{G}$ and $B_{\phi}=480 \mathrm{G}$ (all at $R=25 \mathrm{~cm}$ ), which give the ratio of 0.8 . When this ratio is larger than unity, the inward force to counterbalance the outer ballooning force comes mainly from the toroidal component of internal return current $j_{r t r n \phi}$. Thus the result in figure 5 suggests that when this ratio is near or larger than unity the discharges do not terminate by the blocking of the external return current. Otherwise, the discharges terminate even under finite $B_{Z}$. 


\subsection{Plasma profiles under various $B_{Z}$}

The first series of experiments shows that there are two channels of return path for the vertical charge separation current driven by the electron VTF drift; the internal return path along the helical field lines in the plasma and the external circuit of the conducting vacuum vessel. While this is demonstrated most clearly by the blocking of the return current in the external circuit, such a situation of blocking is unusual. Nowadays the devices usually employ an electrically conducting vacuum vessel to contain the plasmas. Therefore, hereafter we concentrate on the experiments without the blocking.

At this point we shall investigate relationship between the circulation currents and the plasmas in the main room between the top and bottom limiters under various values of $B_{Z}$. We need two dimensional profiles of the electron temperature $T_{e}$, electron density $n_{e}$ and space potential $V_{S}$ in the main room, which constitute the main part of information to study the underlining physics of toroidal ECR plasmas. These data have been obtained by changing the location of the Langmuir probe tip over 169 spatial points $(13$ vertical $\times 13$ radial points) appropriately distributed in the main room on shot by shot basis. In some cases additional 90 points in the shadow area of the limiters are added.

Figure 6 shows the discharges in which $B_{Z}$ is swept slowly from $B_{Z}=6$ G to -6 G. During the initial transition phase of $0.4 \mathrm{~s}$ after breakdown $B_{Z}$ is kept $6 \mathrm{G}$. Then $B_{Z}$ is swept down to $-6 \mathrm{G}$ by using a long sweeping time of $1.5 \mathrm{~s}$ and afterward $B_{Z}$ is kept $-6 \mathrm{G}$. The behavior of the various currents versus $B_{Z}$ is consistent with the current characteristics in figures 3(a) and (b), indicating that the sweeping rate is slow enough for the plasma to be essentially in steady state for each transient $B_{Z}$ field. While all the characteristic currents flowing into the limiters and panels change places between the top and bottom electrodes upon the change of the toroidal field direction, the direction of the toroidal current does not change. On the other hand, while the direction of the toroidal current reversed when the $B_{Z}$ direction is reversed, the characteristic currents flowing into the limiters and panels are symmetric for the direction of $B_{Z}$.

We sweep the probe voltage sawtooth-likely many times during each two-seconds discharge to obtain single probe characteristics within a negligibly small change of $\Delta B_{Z}=0.2 \mathrm{G}$ as shown in figure 7 . Thus the probe data at one fixed spatial point for various $B_{Z}$ from $B_{Z}=-6$ to $6 \mathrm{G}$ is obtained in one discharge.

While figure 7 shows that $T_{e}$ is measured with high accuracy, there is a concern about accuracy of the density measurement since it depends on the validity of effective cross section of the probe surface used for the analysis. Another concern is possible disturbance from the probe insertion deep into the plasma. We check them by comparing the probe result with the interferometer result by using a two-seconds discharge under $B_{Z}=0$. As shown in figure 8, the line-integrated density by the $70 \mathrm{GHz}$ interferometer hardly depends on the Langmuir probe locations except for the initial transition time. Furthermore, it coincides with the line-integrated value estimated by using the probe results along the interferometer chord. 

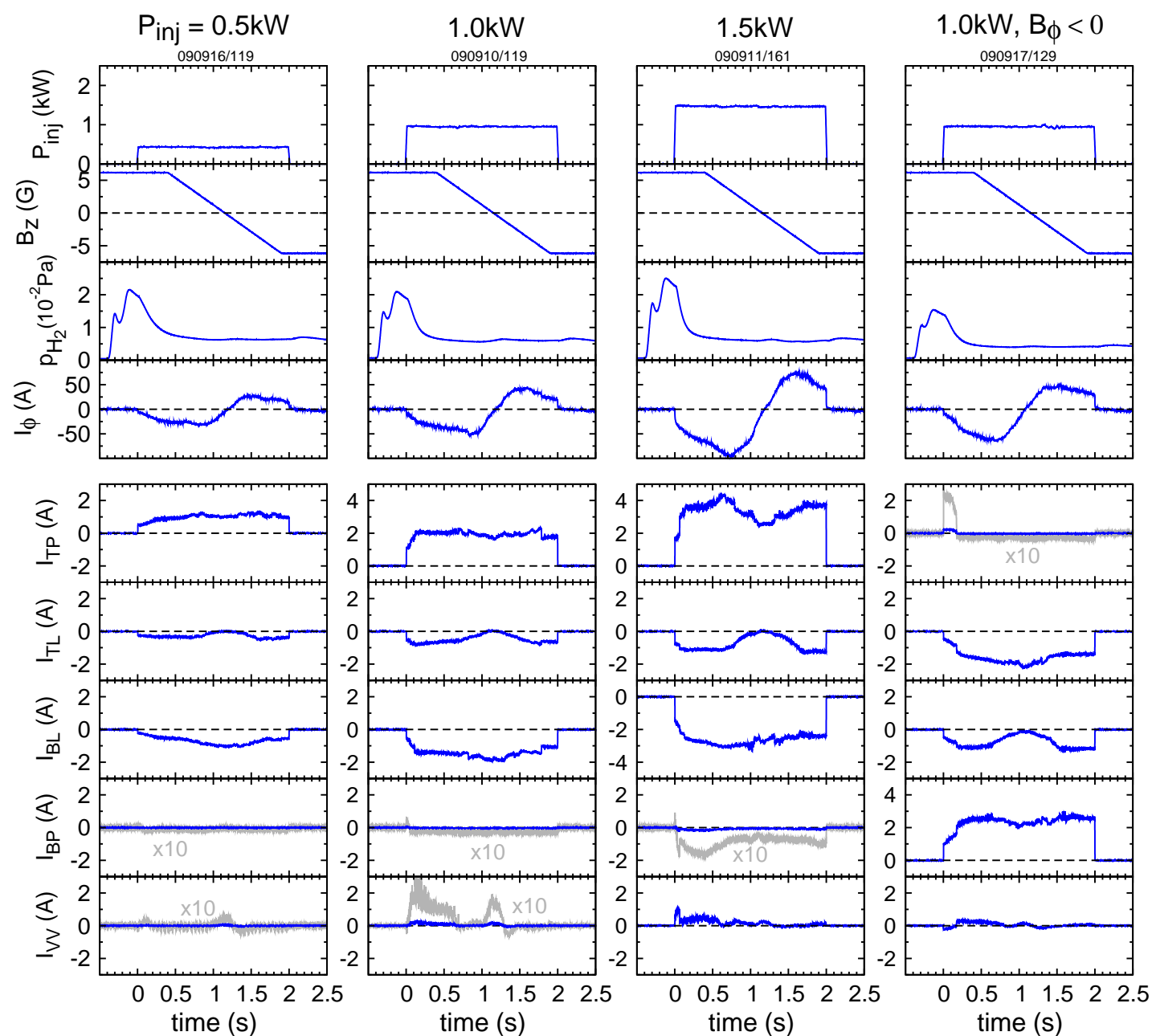

Figure 6. Typical discharges with a $B_{Z}$ sweep for various injection power of microwaves. The right end column is the case with the reversed toroidal field, $B_{\phi}<0$.

Figures 9, 10, 11 and 12 show the profiles of $n_{e}, T_{e}, V_{S}$, and the electron pressure $p_{e}=n_{e} T_{e}$ at $B_{Z}=0,+2,-2,+6,-6 \mathrm{G}$ for the cases of $P_{i n j}=0.5 \mathrm{~kW}$ and $B_{\phi}>0$, $P_{i n j}=1.0 \mathrm{~kW}$ and $B_{\phi}>0, P_{i n j}=1.5 \mathrm{~kW}$ and $B_{\phi}>0$ and $P_{i n j}=1 \mathrm{~kW}$ and $B_{\phi}<0$, respectively. They correspond to the discharges shown in figure 6 .

A number of characteristics are seen from the results in figures $9-12$.

1. The electron temperature $\left(T_{e}\right)$ profiles have a peak slightly outwards from the ECR layer $\left(R_{E C R}=13.7 \mathrm{~cm}\right)$. The peak is extended vertically, making a narrow vertical ridge. This character remains when a weak $B_{Z}$ is applied. The peak value of $T_{e}$ is in the range of $T_{e}=12 \sim 14 \mathrm{eV}$ and does not depend on the microwave injection power in the range of $0.5 \sim 1.5 \mathrm{~kW}$.

2. The electron density $\left(n_{e}\right)$ profiles also have a peak slightly outwards from the ECR layer when $B_{Z}=0$, which is extended vertically, thus making a vertical ridge. The ridge is, however, not quite vertical and somewhat tilts in accordance with the optical image in figure 4(a). The tilt direction is reversed when $\left|B_{Z}\right|$ is increased, which does 

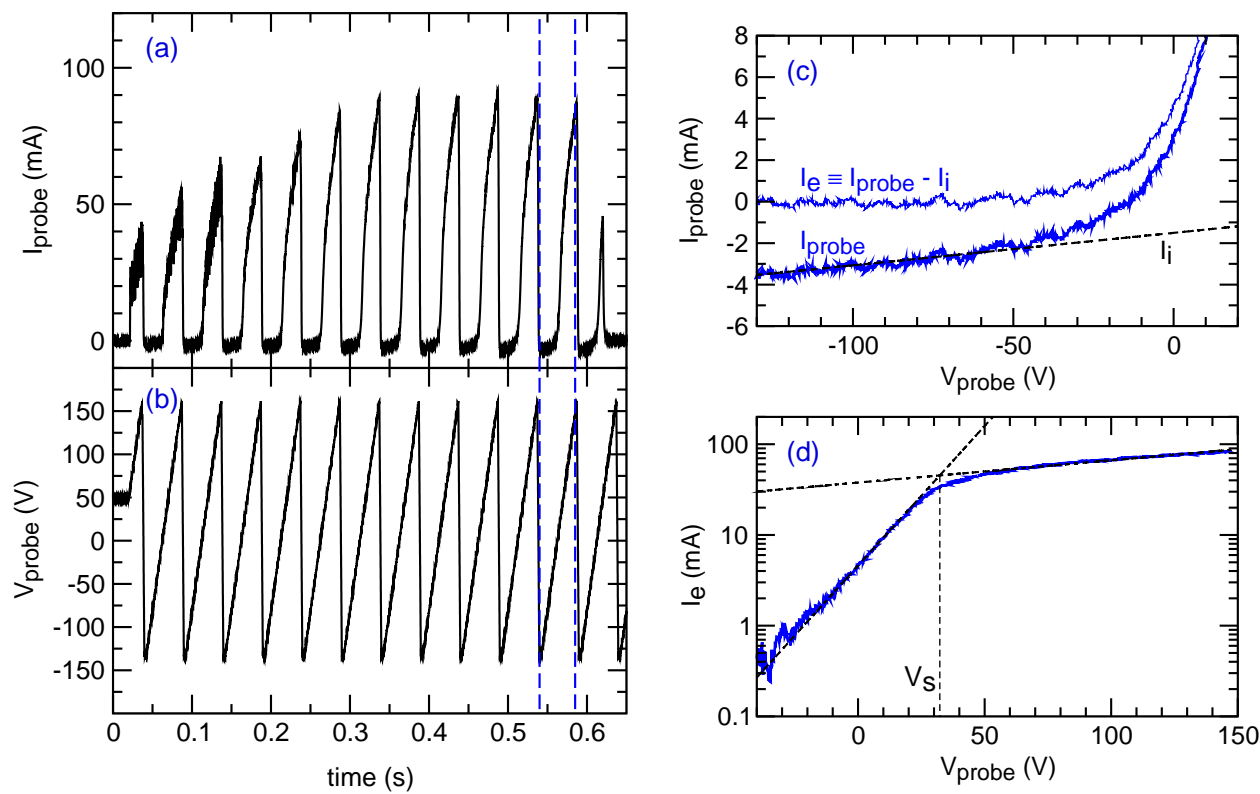

Figure 7. The probe voltage is swept from $-140 \mathrm{~V}$ to $160 \mathrm{~V}$ during $50 \mathrm{~ms}$, which means that 30 sweeps during the Bz sweep from 6 to -6 Gauss in figure 7 . The probe current characteristics between the dotted lines in (a) and (b) are plotted as a function of probe voltage in (c) and (d).
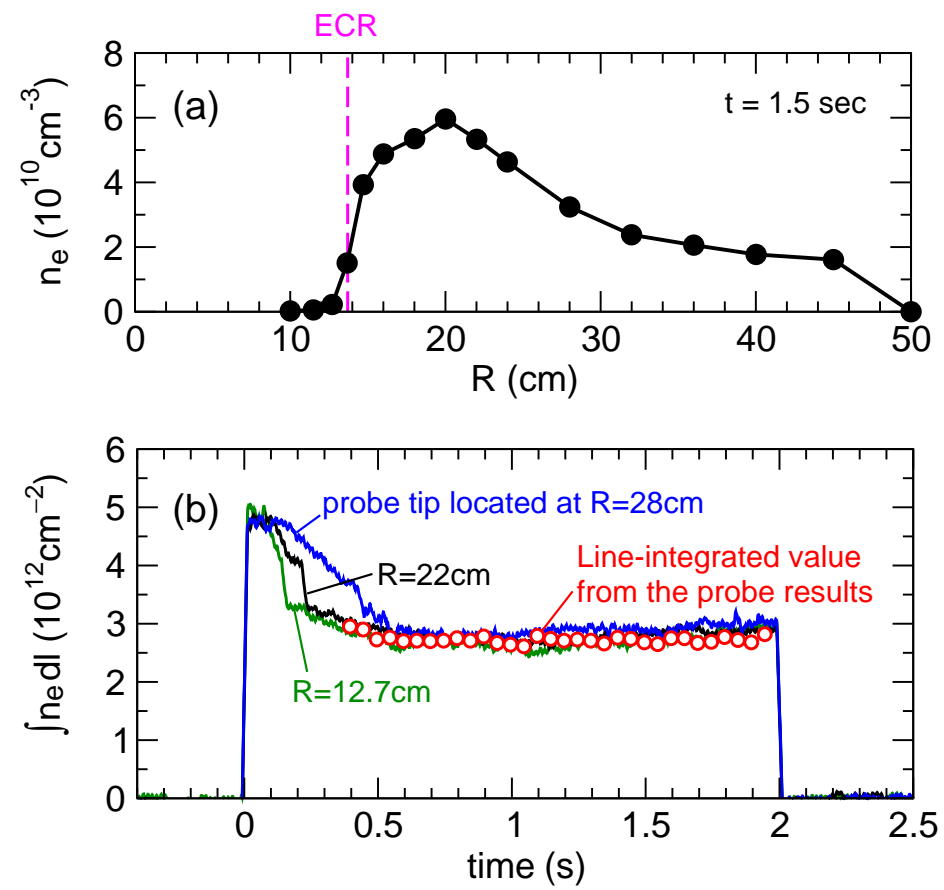

Figure 8. (a) Radial density profile on the mid plane obtained by the Langmuir probe. (b) The line-integrated density by the $70 \mathrm{GHz}$ interferometer hardly depends on the positions of the Langmuir probe on the mid plane except for the initial transition time of discharge. Furthermore, it coincides with the line-integrated value estimated from the probe results along the same chord by assuming that the density profile is axisymmetric. $P_{i n j}=1.5 \mathrm{~kW}$, Hydrogen, $B_{Z}=0.0 \mathrm{G}$ 

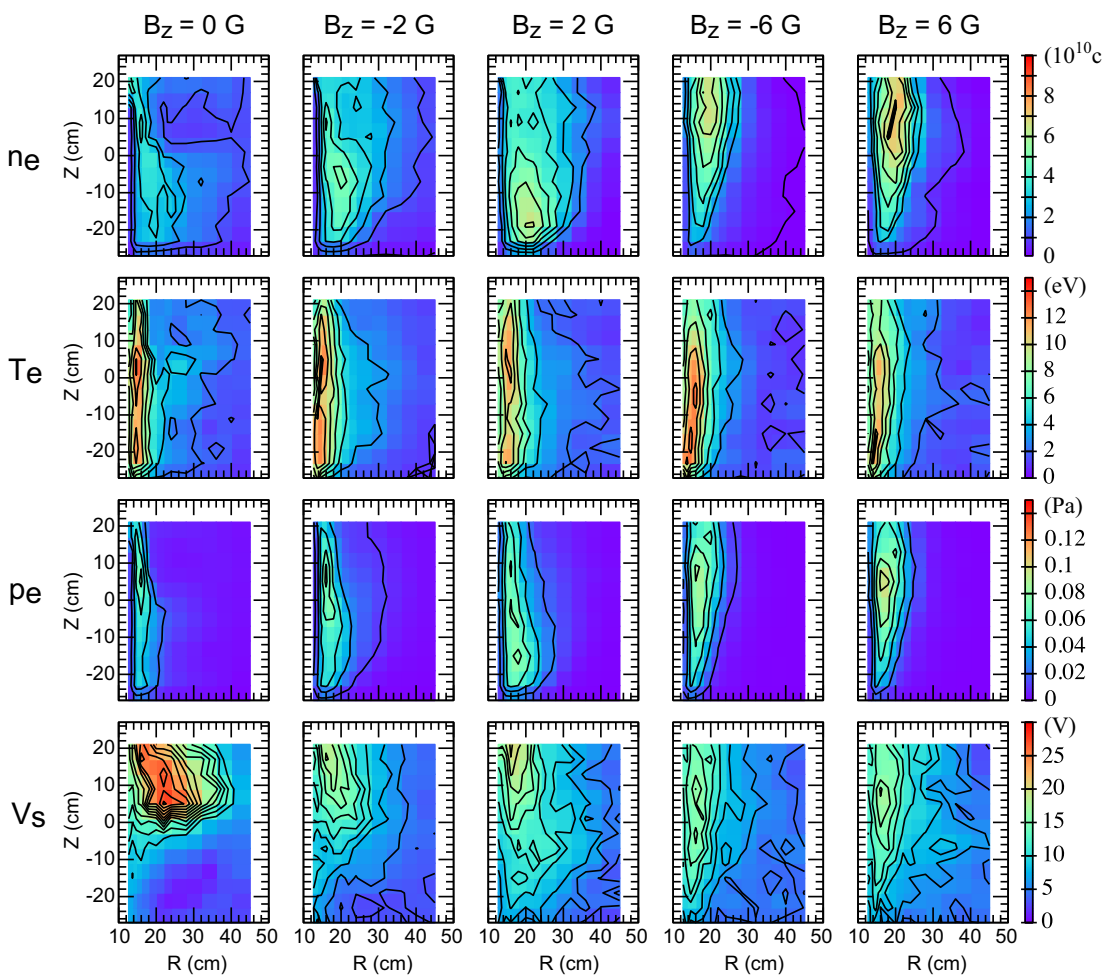

Figure 9. The profiles of $n_{e}, T_{e}, V_{S}$, and $p_{e}=n_{e} T_{e}$ at $B_{Z}=0,+2,-2,+6,-6 \mathrm{G}$ for the cases of $P_{i n j}=0.5 \mathrm{~kW}$. Fundamental ECR layer is located at $R=13.7 \mathrm{~cm}$.
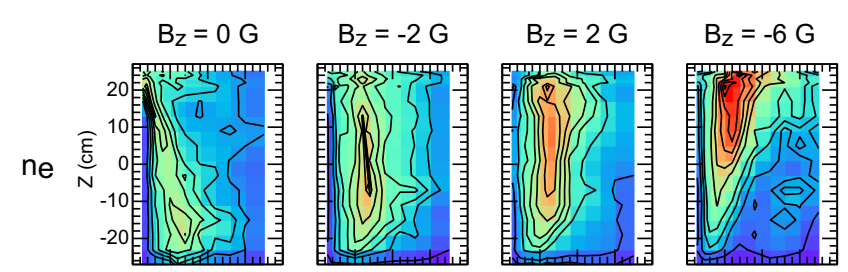

$B_{z}=6 G$
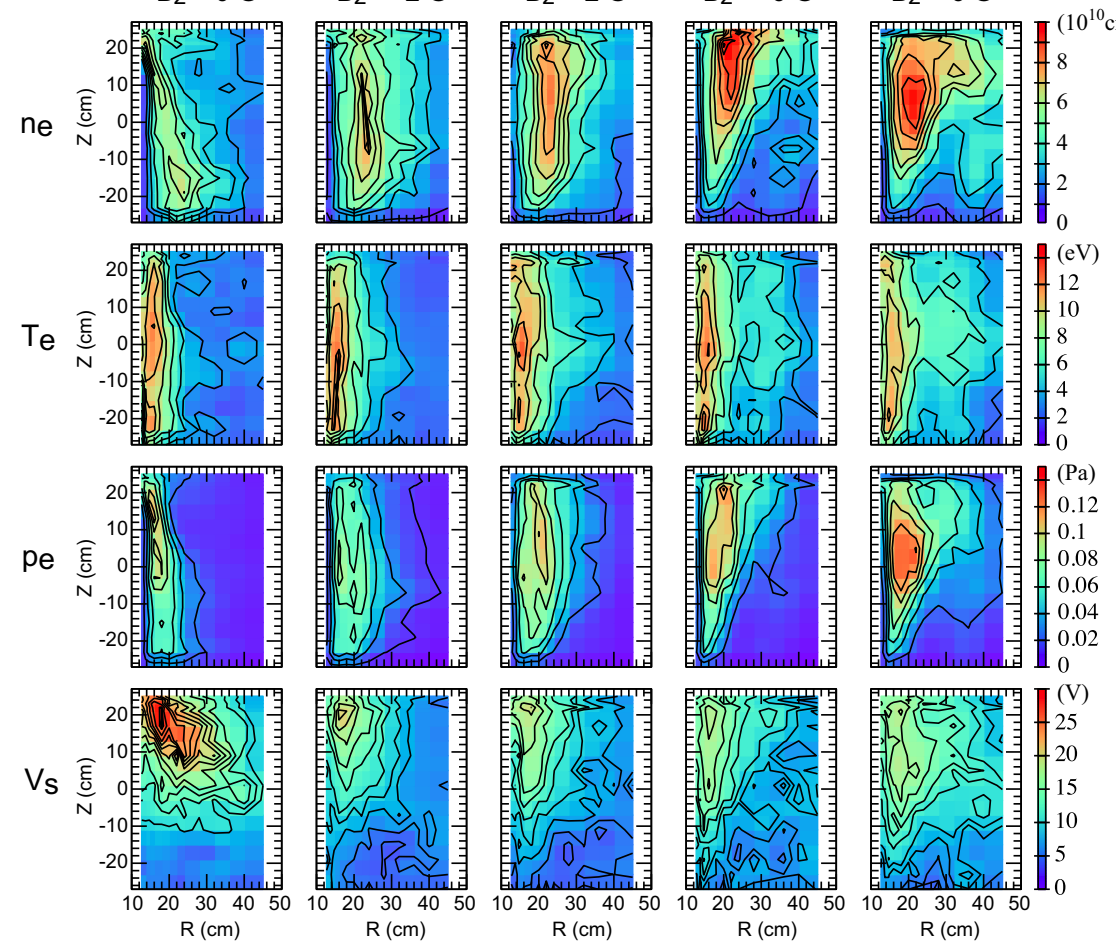

Figure 10. The profiles of $n_{e}, T_{e}, V_{S}$, and $p_{e}=n_{e} T_{e}$ at $B_{Z}=0,+2,-2,+6,-6 \mathrm{G}$ for the cases of $P_{i n j}=1.0 \mathrm{~kW}$. Fundamental ECR layer is located at $R=13.7 \mathrm{~cm}$. 


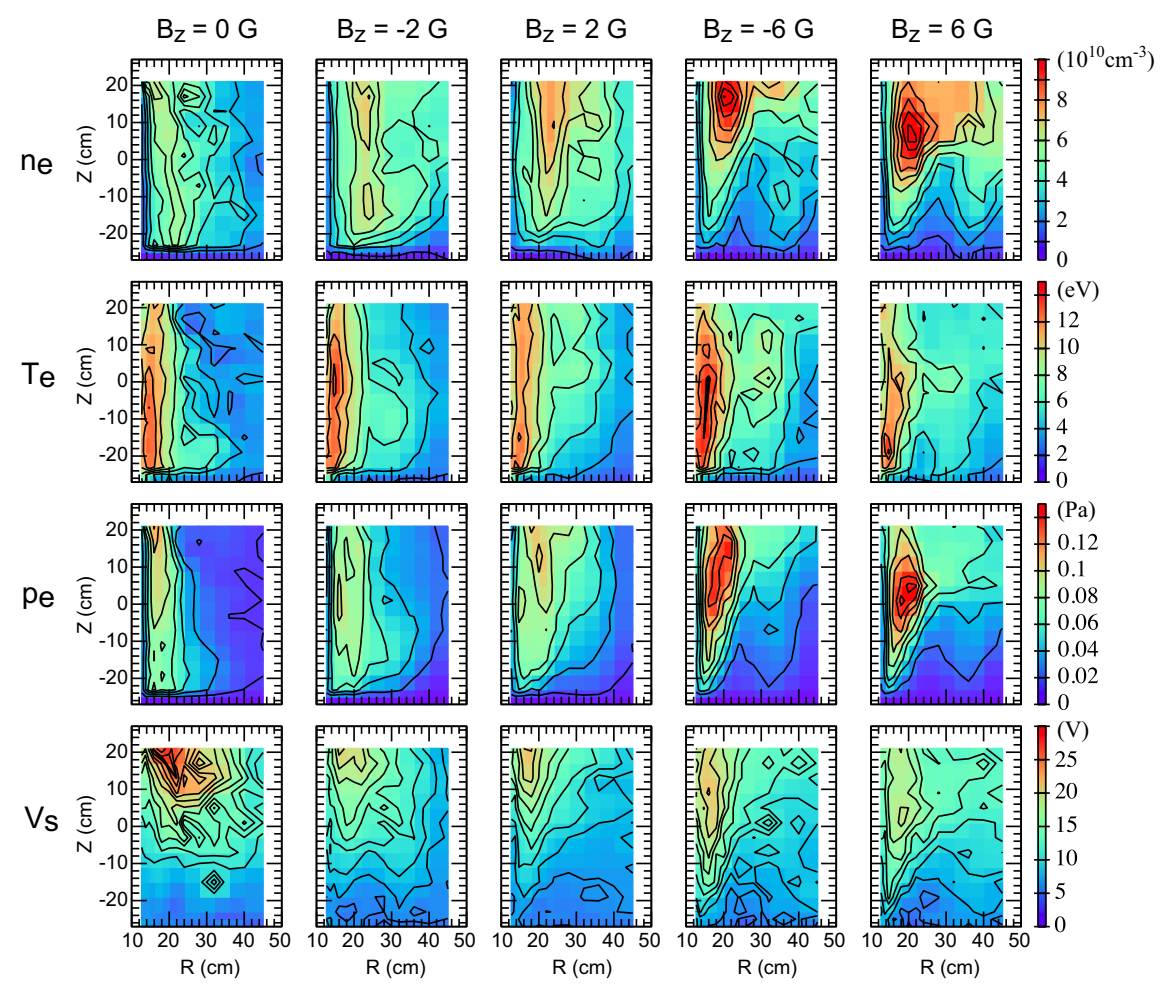

Figure 11. the profiles of $n_{e}, T_{e}, V_{S}$, and $p_{e}=n_{e} T_{e}$ at $B_{Z}=0,+2,-2,+6,-6 \mathrm{G}$ for the cases of $P_{i n j}=1.5 \mathrm{~kW}$. Fundamental ECR layer is located at $R=13.7 \mathrm{~cm}$.

not depend on the sign of $B_{Z}$. As a result the ridge is almost vertical when $\left|B_{Z}\right|=2$ G. In the cases of $B_{Z}=-6$ and $6 \mathrm{G}$, the tilt angle is reversed. More remarkable in these cases with $\left|B_{Z}\right|=6 \mathrm{G}$ is that the density peak increases and shifts upwards, that is, to the ion VTF drift direction, with an expansion of the profile to the low field side. These profiles are reversed or become upside down when the toroidal field is reversed. The peak density is around the cutoff density $\left(n_{c}=7 \times 10^{10} \mathrm{~cm}^{-3}\right.$ for $\left.2.45 \mathrm{GHz}\right)$ in the range from $n_{e}=4 \times 10^{10} \mathrm{~cm}^{-3}$ to $10 \times 10^{10} \mathrm{~cm}^{-3}$ depending on the injected microwave power $P_{i n j}$ and $B_{Z}$ strength. It increases as $P_{i n j}$ and $\left|B_{Z}\right|$ increase.

3. The electron pressure $\left(p_{e}\right)$ profiles also have a peak slightly outwards from the ECR layer when $B_{Z}=0$, which is almost uniformly extended in the vertical direction, thus making a narrow vertical ridge. When $\left|B_{Z}\right|$ increases to $6 \mathrm{G}$, the peak increases and shifts upwards with an expansion of the profile to the low field side, which does not depend on the sign of $B_{Z}$. These profiles are reversed when the toroidal field is reversed.

4. The space potential $\left(V_{S}\right)$ profiles have a hill with a peak near the top electrode (the ion VTF drift side) and just outside the ECR layer. When $B_{Z}=0$, the peak potential is most high $V_{\text {Speak }} \approx 30 \mathrm{~V}$, a few times the $T_{e}$ peak value. The peak potential does not depend on the microwave injection power in the range of $0.5 \sim 1.5 \mathrm{~kW}$. The peak potential decreases as $\left|B_{Z}\right|$ is increased, and is around $V_{\text {Speak }} \approx 20 \mathrm{~V}$ when $\left|B_{Z}\right|=6$ G. The profiles are reversed when the toroidal field is reversed. The above behaviors do not depend on the sign of $B_{Z}$. The peak potential dose not depend on the microwave 

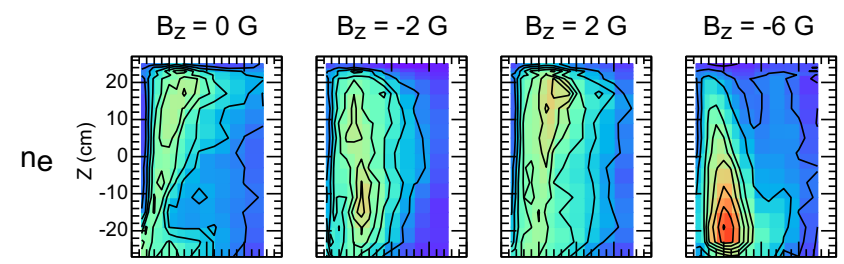

$\mathrm{B}_{\mathrm{z}}=6 \mathrm{G}$
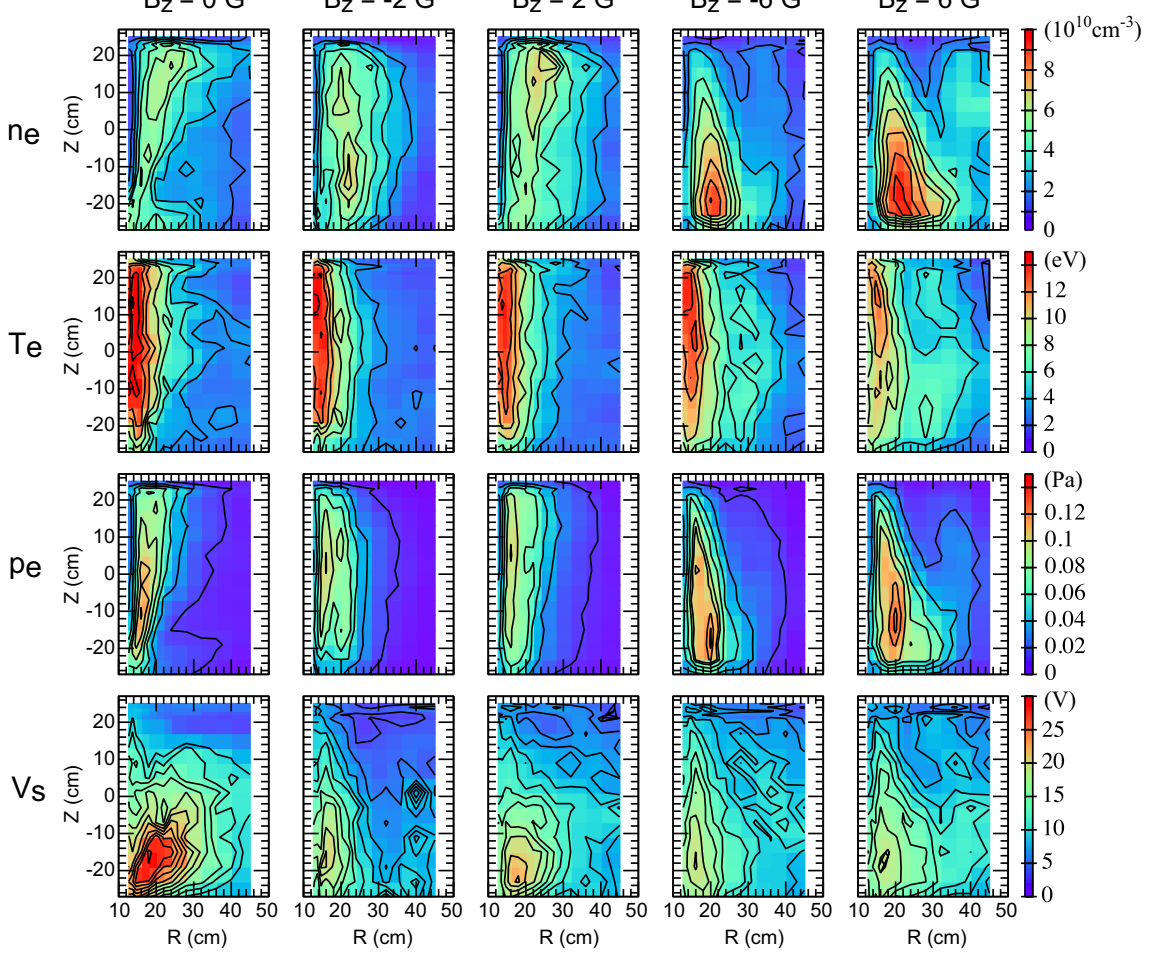

Figure 12. the profiles of $n_{e}, T_{e}, V_{S}$, and $p_{e}=n_{e} T_{e}$ at $B_{Z}=0,+2,-2,+6,-6 \mathrm{G}$ for the cases of $P_{i n j}=1.0 \mathrm{~kW}$. The direction of the toroidal field is reversed, $B_{\phi}<0$. Fundamental ECR layer is located at $R=13.7 \mathrm{~cm}$.

injection power in the range of $0.5 \sim 1.5 \mathrm{~kW}$

5. It is most remarkable that the $n_{e}, P_{e}$ and $V_{S}$ profiles look similar when $\left|B_{Z}\right|=6$ $\mathrm{G}$, suggesting that the electrons are closely coupled along the helical field lines and reach an equilibrium of Boltzmann distribution. They are quite different from the cases with $B_{Z}=0$, where both the electron density and temperature make vertical ridges just outside the ECR layer, while the space potential makes a broad profile with a peak just outside the ECR layer and the peak is extremely shifted to the ion VTF drift side.

\subsection{Plasma profiles with noble gases}

In the third series of experiments we used helium (He), neon (Ne) and argon ( $\mathrm{Ar}$ ) as working gas and compared the results with the cases of hydrogen. Their mass numbers are quite different as shown in table 1 . The procedure of the discharges and the measurements are the same as the second series of experiments. All the characteristics of various currents and the profiles of $n_{e}, T_{e}, V_{S}$ and $p_{e}$ versus $B_{Z}$ are qualitatively similar to the case of hydrogen gas described in section 3.2. Therefore, only the profiles at $B_{Z}=0$ and $B_{Z}=6 \mathrm{G}$ are shown in figures 13 and 14 , respectively. While the statements 1-5 for the hydrogen case are also applicable for the present case of noble gases, some features are still seen from the profiles in figures 13 and 14 .

1. The space potential in the case of $B_{Z}=0$ decreases monotonically as the mass 

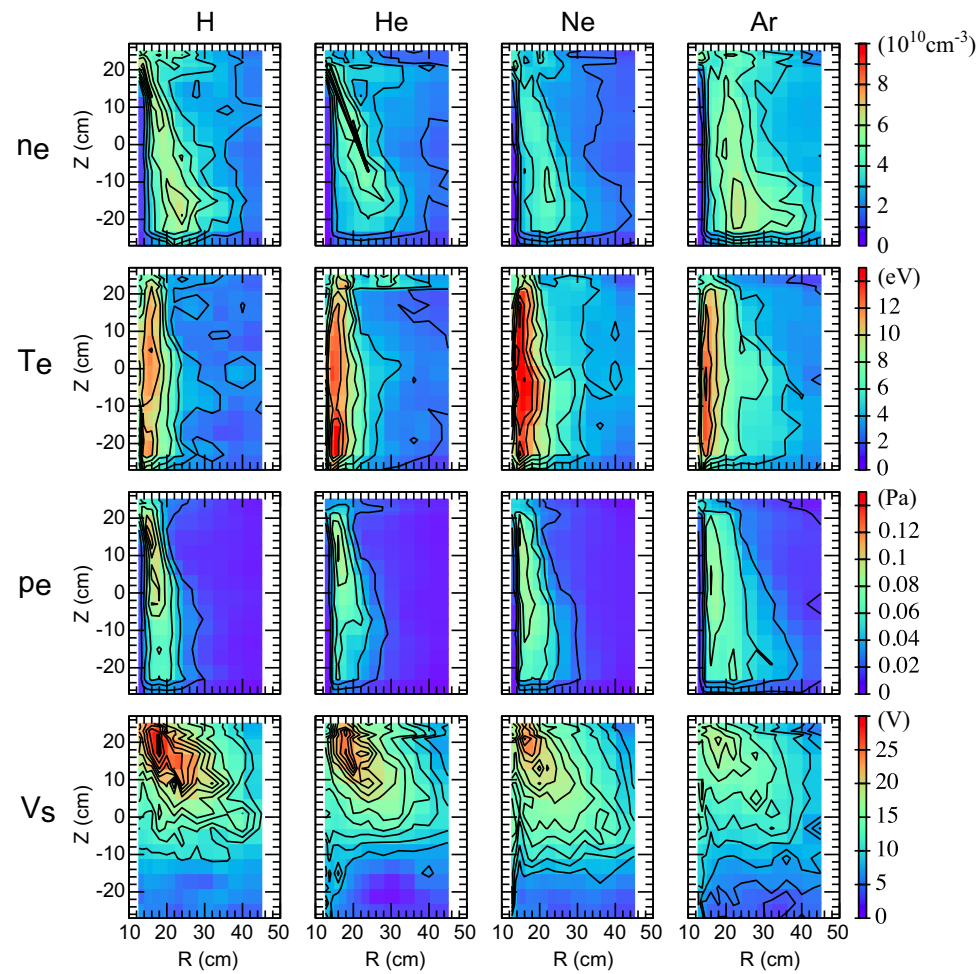

Figure 13. Various profiles under no $B_{Z}$ in the case of noble gasses of $\mathrm{He}, \mathrm{Ne}$ and Ar. The profiles for the hydrogen case are also plotted for comparison. $P_{i n j}=1 \mathrm{~kW}$.
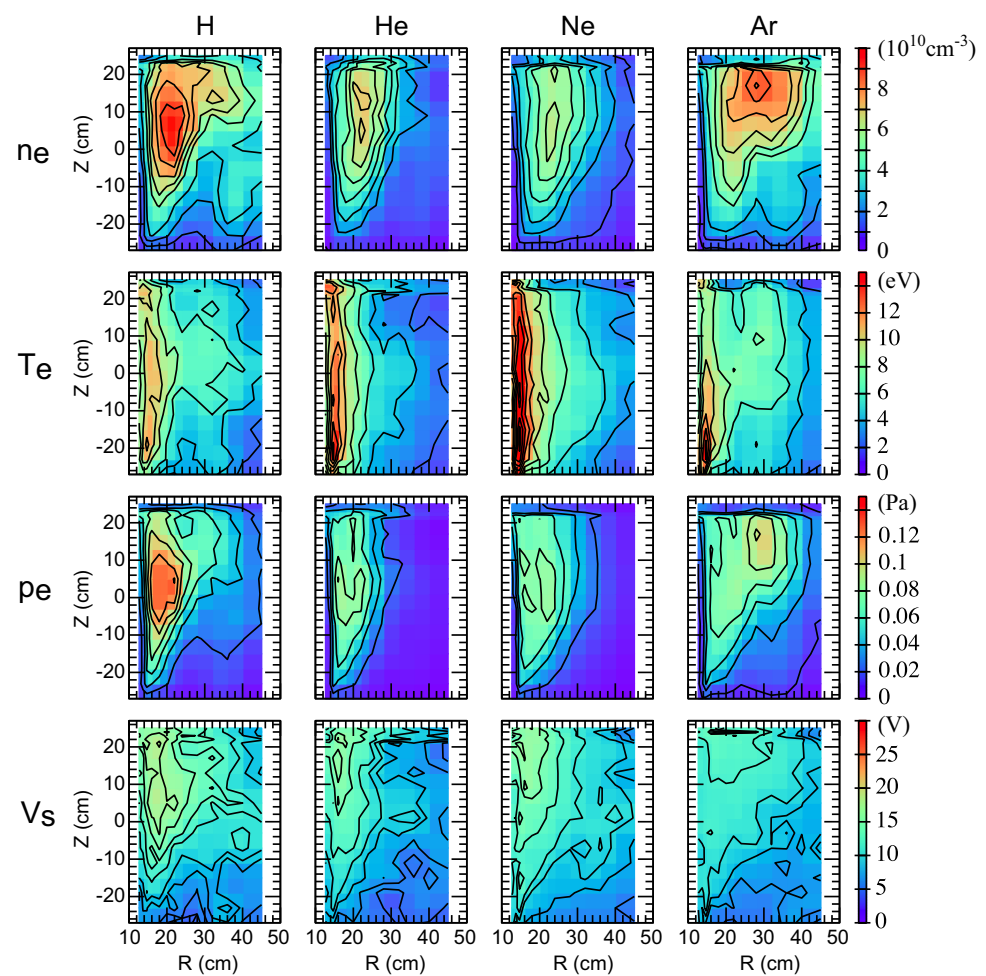

Figure 14. Various profiles under $B_{Z}=6 \mathrm{G}$ in the case of noble gasses of $\mathrm{He}, \mathrm{Ne}$ and Ar. The profiles for the hydrogen case are plotted for comparison. $P_{i n j}=1 \mathrm{~kW}$. 
Table 1. Mass numbers and ionization energies of $\mathrm{H}, \mathrm{He}, \mathrm{Ne}$ and $\mathrm{Ar}$

\begin{tabular}{|c|c|c|c|c|}
\hline Items & $\mathrm{H}$ & $\mathrm{He}$ & $\mathrm{Ne}$ & $\mathrm{Ar}$ \\
\hline Mass number & 1 & 4 & 20 & 40 \\
\hline 1st ionization energy (eV) & 13.6 & 24.6 & 21.6 & 15.8 \\
\hline 2nd ionization energy (eV) & & 54.4 & 41.0 & 27.6 \\
\hline
\end{tabular}

number increases, although the peak electron temperature does not change. It is in the range of $T_{e}=12 \sim 14 \mathrm{eV}$. The potential peak value is $V_{\text {Speak }} \simeq 20 \mathrm{~V}$ in the case of argon while it is $30 \mathrm{~V}$ in the case of hydrogen. There is, however, no change in its profile pattern.

2. The electron density profiles in the case of argon plasma expand significantly outwards compared with the other cases.

3. The electron densities in the hydrogen and argon plasmas are higher than those in the helium and neon plasmas. This may be ascribed to the lower ionization energy of these gases (see table 1)

4. Especially, the electron density in the argon plasmas at $B_{Z}=6 \mathrm{G}$ is higher than the cutoff density $\left(7 \times 10^{10} \mathrm{~cm}^{-3}\right)$ over a broad area at the upper section. The electron temperature at the ECR layer just behind of this broad cutoff area is lower than that at the lower half section. It looks like that the broad cutoff area intercepts the penetration of microwave power to the behind ECR layer. Corresponding to this feature, the electron temperature at the ECR layer in the neon plasma, in which there is no cutoff area, is significantly high, $T_{e} \simeq 14 \mathrm{eV}$. The presence of the broad overdense area at the lower field side from the ECR layer in the argon case suggests that some portion of microwave power is absorbed via the mode-conversion to the electron Berstein waves at the upper hybrid resonance layer.

\section{Analyses and Discussions}

\subsection{Mirror symmetries for external coil currents}

Schematic illustrations in figure 15 show that the various profiles in figures $9-12$ have the mirror symmetry for the reversal of the toroidal field coil current as well as the mirror symmetry for the reversal of the vertical field coil current. For example, the reversal of the toroidal field coil current from figure 15 (a) to (b) is equivalent to taking the mirror image of figure 15(a) with a flat mirror between figures 15 (a) and (b). Then all the plasma profiles should become upside down when the direction of the toroidal field coil current is reversed. This is indeed the case as observed for the profiles in figure $10\left(B_{\phi}>0\right.$ and $\left.P_{i n j}=1 \mathrm{~kW}\right)$ and figure $12\left(B_{\phi}<0\right.$ and $\left.P_{i n j}=1 \mathrm{~kW}\right)$. In the case of a vertical mirror parallel to the Z-axis between figures 15(c) and (e), there arise no change in mirror images of the various plasma profiles if they are axisymmetric 
around the Z-axis, while the direction of the vertical field coil current is reversed in the mirror image. This is the case as seen in figures $9-12$, suggesting that the profiles are axisymmetric.

The small tilt of the narrow vertical ridge of the electron density in the case of $B_{Z}=0$ has a mirror symmetry for the toroidal coil current. This tilt disappears when $B_{Z}=-2 \mathrm{G}$ as well as when $B_{Z}=2 \mathrm{G}$. These results suggest that this tilt of density profile in the case of $B_{Z}=0$ is not due to some error fields and, therefore, it reflects essential features of the ECR plasmas. The same statement may be applied to the other profiles, especially to the profile of the space potential.

The toroidal plasma current appears when $B_{Z}$ is finite and the current changes its direction when $B_{Z}$ is reversed, which is in accordance with the mirror symmetry.

(a)

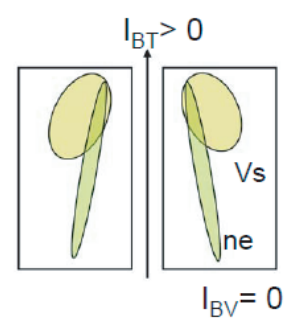

Mirror surface

(b)

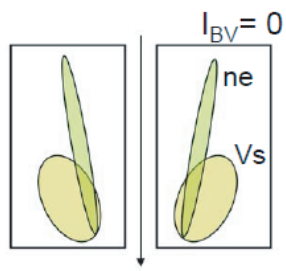

$\mathrm{I}_{\mathrm{BT}}<0$

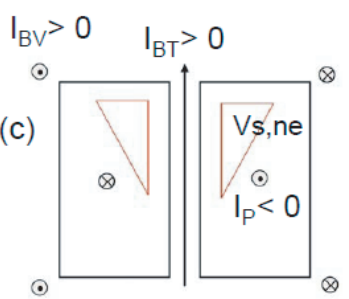

Mirror surface

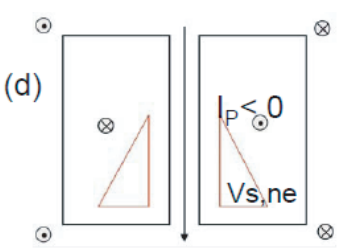

$\mathrm{I}_{\mathrm{BV}}>0 \quad \mathrm{I}_{\mathrm{BT}}<0$
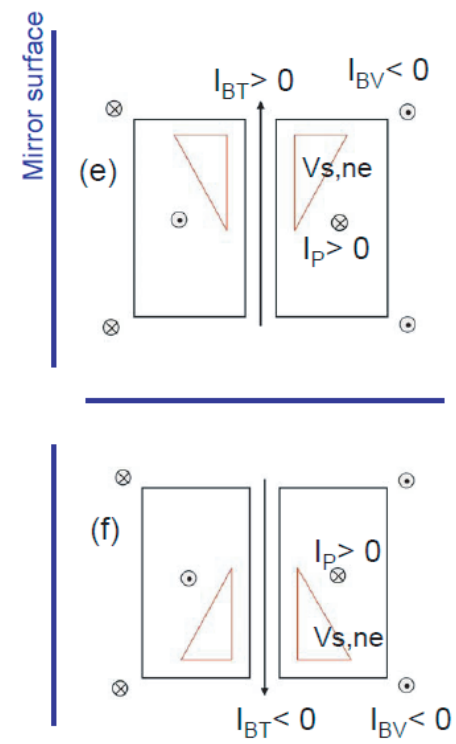

Figure 15. Schematic illustrations of the various profiles in figures $9-12$ and the direction of toroidal plasma current for various combinations of directions of the vertical field coil current $I_{B V}$ and the toroidal field coil current $I_{B T}$. They have the mirror symmetry for the reversal of $I_{B V}$ as well as the mirror symmetry for the reversal of $I_{B T}$.

\subsection{Space and time scales of various processes}

Typical parameters of the discharges and plasmas in figures $9-12$ are followings; $n_{e} \approx 7 \times 10^{16} \mathrm{~m}^{-3}, T_{e} \approx 10 \mathrm{eV}, V_{S} \approx 30 \mathrm{~V}, E \approx V_{S} /$ plasma radius $\approx 30 \mathrm{~V} / 0.2 \mathrm{~m}=150$ $\mathrm{Vm}^{-1}, p_{H 2} \approx 4 \times 10^{-3} \mathrm{~Pa}, n_{H 2} \approx 1.1 \times 10^{18} \mathrm{~m}^{-3}$, and $B_{\phi}=0.0875 \mathrm{~T}$ at the ECR layer $R_{E C R}=0.137 \mathrm{~m}$. By using these values various parameters and characteristics are estimated as follows.

The electron pressure is $p_{e} \equiv n_{e} T_{e} \approx 0.1 \mathrm{~Pa}$ and the magnetic pressure is $p_{\text {mag }} \equiv B_{\phi}^{2} / 2 \mu_{0} \sim 10^{3} \mathrm{~Pa}$. 
The VTF drift velocity of thermal electrons is typically,

$$
V_{V T F} \approx \frac{2 T_{e}}{e} \frac{1}{R B_{\phi}} \approx 1700 \mathrm{~ms}^{-1} .
$$

When we used the strength of toroidal field at $R=0.16 \mathrm{~m}$, the $\mathbf{E} \times \mathbf{B}$ drift velocity is typically,

$$
V_{E \times B}=E / B \approx 150 / 0.075 \approx 2000 \mathrm{~ms}^{-1},
$$

being nearly the same velocity as the electron VTF drift velocity. Then, the time needed for thermal electrons to traverse the plasma cross section is typically,

$$
\tau_{\text {trv }}=\frac{\text { plasma radius }}{V_{\text {drift }}} \approx \frac{0.2}{1700} \approx 0.12 \mathrm{~ms},
$$

where $V_{\text {drift }}=V_{V T F}$ or $V_{E \times B}$. The electron-ion collision time for thermal electrons and protons is $\tau_{e i} \approx 0.01 \mathrm{~ms}$, which is much shorter than the traverse time.

The ionization rate is typically, $<\sigma_{i o n} v>\approx 5.0 \times 10^{-15} \mathrm{~m}^{-3} \mathrm{~s}^{-1}$, where $<>$ indicates the quantity averaged over the electron velocity distribution. Then, the ionization time, which is the typical time needed for an electron to make an ionization collision with a hydrogen atom, is defined by,

$$
<\tau_{\text {ion }}>\equiv \frac{1}{n_{H 2}<\sigma_{i o n} v>} \approx 0.17 \mathrm{~ms} .
$$

This is nearly equal to the traverse time. The recombination rate is typically, $\left\langle\sigma_{r e c} v>\approx\right.$ $8 \times 10^{-20} \mathrm{~m}^{-3} \mathrm{~s}^{-1}$. Then, the recombination time is

$$
<\tau_{\text {rec }}>\equiv \frac{1}{n_{i}<\sigma_{\text {rec }} v>} \approx 180 \mathrm{~s},
$$

where $n_{i}=n_{e}$ is assumed. This is $\sim 10^{3}$ times longer than the traverse time. This result and the result that the traverse time is nearly equal to the ionization time suggest that the plasma is maintained by the balance between the ionization events in the plasma and the recombination events that take place when plasma particles reach the solid wall.

The divergence of the electrostatic field is typically, $\nabla \cdot \mathbf{E} \approx E /($ plasma radius $) \approx$ $750 \mathrm{Vm}^{-2}$. Then, typical net charge density is estimated to be $\rho \equiv \epsilon_{0} \nabla \cdot \mathbf{E} \approx$ $7 \times 10^{-9} \mathrm{Cm}^{-3}$. The ratio to the charge density of plasma electrons is estimated to be,

$$
\frac{\rho}{e n_{e}} \approx \frac{7 \times 10^{-9}}{1.6 \times 10^{-19} \times 7 \times 10^{16}} \approx 7 \times 10^{-7},
$$

, indicating that level of charge neutrality is quite high.

\subsection{Fluid description}

The momentum balance equations of the electron fluid and ion fluid in a steady state [21] are, respectively,

$$
\begin{aligned}
& m n_{e}\left(\mathbf{u}_{e} \cdot \nabla\right) \mathbf{u}_{e}=-e n_{e}\left(\mathbf{E}+\mathbf{u}_{e} \times \mathbf{B}\right)-\nabla p_{e}+\mathbf{R}_{e i} \\
& M n_{i}\left(\mathbf{u}_{i} \cdot \nabla\right) \mathbf{u}_{i}=e n_{i}\left(\mathbf{E}+\mathbf{u}_{i} \times \mathbf{B}\right)-\nabla p_{i}+\mathbf{R}_{i e}
\end{aligned}
$$


where $\mathbf{R}_{e i}=-\mathbf{R}_{i e}=m n_{e}<\nu_{e i}>\left(\mathbf{u}_{i}-\mathbf{u}_{e}\right)$ describes the collisional momentum transfer between the electrons and ions. Here we assume single species of ion and that they are singly ionized. Furthermore we assume that ion temperature is much lower than the electron temperature, say $T_{i} \approx 1 \mathrm{eV}$, since there is no direct heating mechanism for ions in the present low density ECR plasmas. Then the above equations may be further simplified by estimating each term by usage of the parameters described in section 4.2 and dropping small terms compared with the leading terms.

The simplified equations for the momentum balance perpendicular to the magnetic field are,

$$
\begin{array}{ll}
0=e n_{e}\left(\mathbf{E}+\mathbf{u}_{e} \times \mathbf{B}\right)+\nabla p_{e} & \text { for the electron fluid } \\
0=\mathbf{E}+\mathbf{u}_{i} \times \mathbf{B} & \text { for the ion fluid }
\end{array}
$$

Where $\mathbf{E}=-\nabla V_{S}(R, Z)$ is the electrostatic field.

From equations (8) and (9) we obtain the equation for local force balance in the perpendicular direction.

$$
\mathbf{j} \times \mathbf{B}=\nabla p_{e}, \text { where } \mathbf{j} \equiv e n_{e}\left(\mathbf{u}_{i}-\mathbf{u}_{e}\right)
$$

This equation is written in terms of the current density, magnetic field and electron pressure (we neglect ion pressure compared with electron pressure), and therefore, is relevant for analysis of the experimental results. The radial and vertical components of the above local equilibrium equation are;

$$
\begin{array}{ll}
R-\text { component }: & j_{\phi} B_{Z}-j_{Z} B_{\phi}=\partial p_{e} / \partial R \\
Z \text {-component }: & j_{R} B_{\phi}-j_{\phi} B_{R}=\partial p_{e} / \partial Z
\end{array}
$$

The equations (11) and (12) with the constraint for the current

$$
0=\nabla \cdot \mathbf{j}=\frac{1}{R} \frac{\partial\left(R j_{R}\right)}{\partial R}+\frac{\partial j_{Z}}{\partial Z}
$$

constitute the basic set of equations to analyze various equilibrium characteristics of the present axisymmetric plasmas.

By substituting the following relationships from equations (11) and (12),

$$
j_{R}=\frac{1}{B_{\phi}}\left(j_{\phi} B_{R}+\frac{\partial p_{e}}{\partial Z}\right) \text { and } j_{Z}=\frac{1}{B_{\phi}}\left(j_{\phi} B_{Z}-\frac{\partial p_{e}}{\partial R}\right)
$$

into equation (13), we have

$$
\begin{aligned}
0 & =\frac{1}{R} \frac{\partial}{\partial R}\left[\frac{R}{B_{\phi}}\left(j_{\phi} B_{R}+\frac{\partial p_{e}}{\partial Z}\right)\right]+\frac{\partial}{\partial Z}\left[\frac{1}{B_{\phi}}\left(j_{\phi} B_{Z}-\frac{\partial p_{e}}{\partial R}\right)\right] \\
& =\frac{1}{B_{\phi}}\left[\frac{2}{R}\left(j_{\phi} B_{R}+\frac{\partial p_{e}}{\partial Z}\right)+\frac{\partial\left(j_{\phi} B_{R}\right)}{\partial R}+\frac{\partial j_{\phi}}{\partial Z} B_{Z}+j_{\phi} \frac{\partial B_{Z}}{\partial Z}\right] \\
& \simeq \frac{1}{B_{\phi}}\left(\frac{2}{R} \frac{\partial p_{e}}{\partial Z}+\frac{\partial j_{\phi}}{\partial Z} B_{Z}\right)
\end{aligned}
$$

Here, we neglect the terms with $B_{R}$ and $\partial B_{Z} / \partial Z$. Then the equation is reduced to

$$
0=\frac{\partial}{\partial Z}\left[\frac{2}{R} p_{e}(R, Z)+B_{Z} j_{\phi}(R, Z)\right]
$$


The solution is

$$
R B_{Z} j_{\phi}(R, Z)=-2\left[p_{e}(R, Z)-p_{0}(R)\right],
$$

where $p_{0}$ is a constant of integration along the Z-coordinate at a fixed $R$ point and, therefore, it is a function of $R$.

\subsection{Equilibrium and current circulation under no $B_{Z}$}

When $B_{Z}=0$, equation (14) reduces to

$$
p_{e}(R, Z)=p_{0}(R)
$$

This result [8] predicts that the pressure profile is uniform along the $\mathrm{Z}$ direction. The pressure profiles in the $B_{Z}=0$ case in figures $9-13$ are roughly consistent with this prediction. When $B_{Z}=0$ the total vertical current $I_{Z}$ that flows across a horizontal plane at $\mathrm{Z}$ is estimated from the fluid equation (11) as

$$
\begin{aligned}
I_{Z}(Z) & \equiv \int j_{Z}(R, Z) 2 \pi R d R=-\int \frac{\partial p_{e}}{\partial R} \frac{2 \pi R}{B_{\phi}} d R \\
& =\frac{4 \pi}{R B_{\phi}} \int R p_{e}(R, Z) d R .
\end{aligned}
$$

Here,

$$
j_{Z}(R, Z)=-\frac{1}{B_{\phi}} \frac{\partial p_{e}(R, Z)}{\partial R}
$$

is the vertical component of the diamagnetic drift current. It is also possible to obtain the vertical current by the drift orbit picture. The electrons drift by the VTF drift and by the $\mathbf{E} \times \mathbf{B}$ drift while the ions drift only by the $\mathbf{E} \times \mathbf{B}$ drift. Net contribution to current comes from the electron VTF drift. The same result as equation (16) is obtained by summing up the contribution from the VTF drift of each electron over Maxwell velocity distribution (see equation (2)). This indicates that the current given by equation (16) is the electron charge separation current that originates from the VTF drift of electrons. Equivalence of both pictures in the case of a simple toroidal plasma is explicitly shown in a standard textbook [22]. The book shows that the accumulation rate at the top of the plasma or, in other words, the local current density flowing into the top (or bottom) electrode is the same as that by the VTF model and given by

$$
j_{Z T O P}(R)=j_{V T F e}(R)=\frac{2 p_{e}(R)}{R B_{\phi}} .
$$

We present two models for the vertical current density, the diamagnetic drift current predicted by the fluid picture, equation (17), and the VTF-drift charge separation current, equation (2). The former is valid. While the latter is not quite valid as the formula for the local current density, it still gives the correct local charge accumulation rate at the top and bottom of the plasma [22]. The point is that both models give the same local charge accumulation rate. Therefore, equation (3) gives the valid toroidal current density as the internal return current of the vertical charge separation current. 
We used equation (2) for convenience since the VTF-drift picture gives more directly the charge accumulation rate, equation (18), than the fluid picture.

By using the data of electron pressure profiles in figures $9-11$, we calculate the total vertical current $I_{Z}$ as a function of $Z$ by using equation (16). The results are plotted in figure 16, where they are compared with the electrode currents for three cases of injection power of $P_{i n j}=0.5,1.0$ and $1.5 \mathrm{~kW}$. In all cases the vertical charge separation current integrated over any horizontal plane in the plasma $\left(I_{Z}(\mathrm{Z})\right)$ is nearly equal to both electrode currents at the top and bottom, indicating that the charge separation current driven by the ECR heated electrons circulates smoothly via the external circuit. That $I_{Z}$ hardly depends on $Z$ in figure 16 indicates that the current direction is almost vertical and the radial component of the current toward the vessel wall is negligibly small.
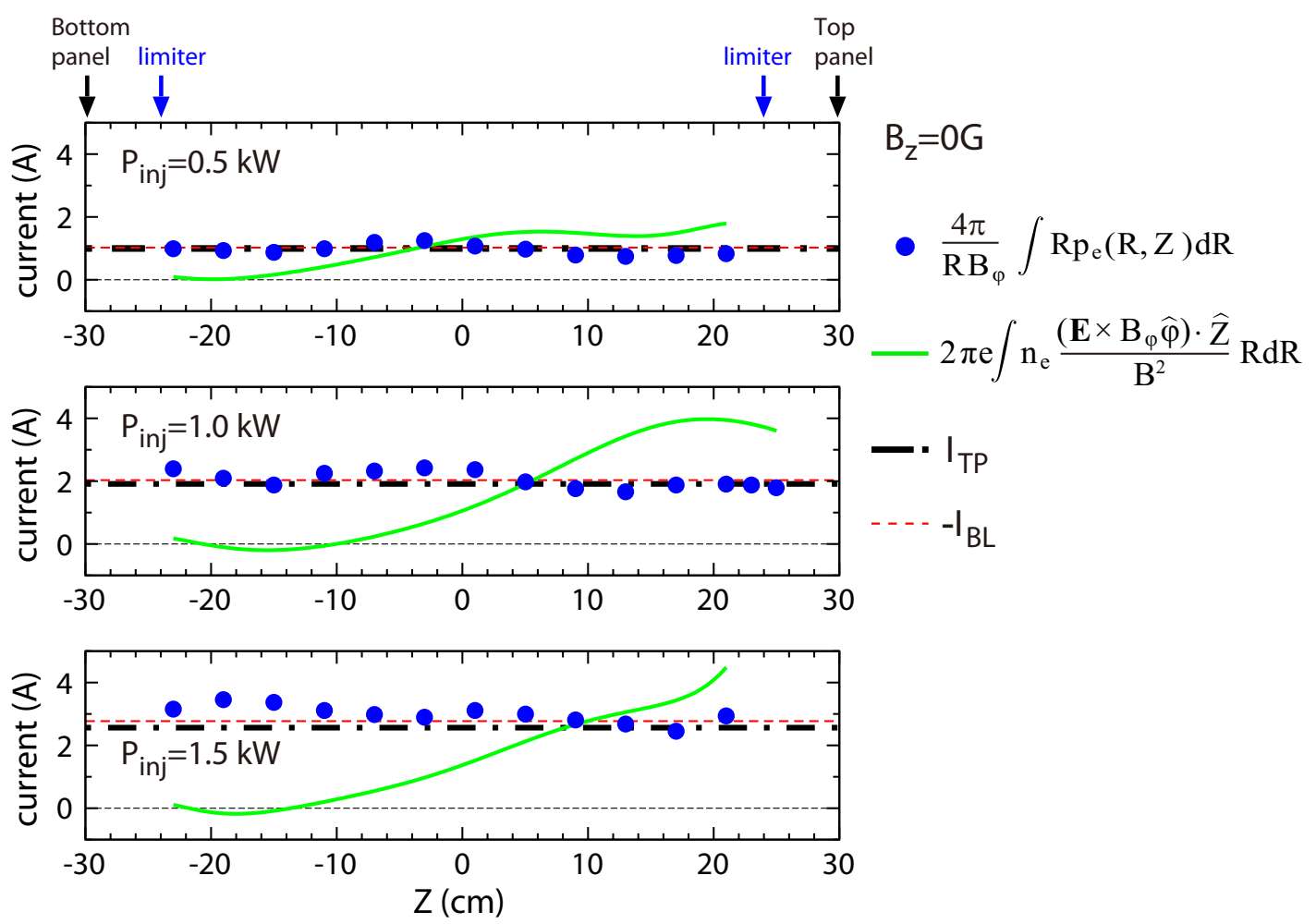

Figure 16. Vertical components of diamagnetic current (solid circles) and $\mathbf{E} \times \mathbf{B}$ current (green lines) that flow across horizontal planes at various vertical locations for the cases of $P_{i n j}=0.5,1.0$ and $1.5 \mathrm{~kW}$. The results are obtained by using the profiles in figures $9-11$.

Thus, the pressure profile predicted by equation (15) reflects the constraint of $\nabla \cdot \mathbf{j}=0$ on the VTF-drift current of electrons under this special case of $B_{Z}=0$, where there is no other return path. This constraint is well satisfied in the present case of long wavelength wave at $2.45 \mathrm{GHz}$ in the small device. Suppose the opposite case in large devices for magnetic fusion, where the injected microwave beam is well localized. How does the discharge stability change when the heating zone changes along the vertical 
coordinate of the ECR layer? In other words, which is more favorable to satisfy the constraint among the top, mid and bottom heating. Under a sufficiently strong $B_{Z}$ there arises the second return path along the helical field line and the restriction on the pressure profile is removed and the density profile may be relaxed to the slow ion VTF drift. Actually the pressure profile shifts to the ion VTF drift side and expands outward as shown in figures $9-12$.

The result shown in figure 16 suggests that the ion contribution is negligible in the vertical charge separation current in the main room. This seems reasonable since the ion pressure may be very low in the present low density ECR-heated plasmas. On the other hand, the same amount of ion current is observed to flow into the top panel as shown in figure 3(a). A possible process that may resolve this puzzle is as follows.

First we consider the problem by using the particle orbit picture. As shown in figures $9-11$ when $B_{Z}=0$ there arises a potential hill with a peak near the top electrode and just outside the ECR layer. Since all electrodes and vessel walls that surround the plasma have essentially ground potential, nested equipotential contours are formed around the peak. Thus charged particles circulate around the peak by $\mathbf{E} \times \mathbf{B}$ drift along the equipotential contours in addition to the VTF drift. While the electrons drift by the VTF drift in addition to the $\mathbf{E} \times \mathbf{B}$ drift, the ions drift essentially only by the $\mathbf{E} \times \mathbf{B}$ drift since the ion temperature would be quite lower than the electron temperature and their VTF drift could be neglected except for the regions near the electrodes where they may have significant kinetic energy. While this $\mathbf{E} \times \mathbf{B}$ drift does not generate net current since the $\mathbf{E} \times \mathbf{B}$ drift velocity is the same for all charged particles, ions can reach the region between the potential hill and the top electrode by the $\mathbf{E} \times \mathbf{B}$ circulation.

We use the fluid equations to estimate quantitatively amount of the $\mathbf{E} \times \mathbf{B}$ flux. The solutions for the two fluid equations (8) and (9) are, respectively, given by

$$
\begin{aligned}
& \mathbf{u}_{i}=\frac{\mathbf{E} \times \mathbf{B}}{B^{2}} \equiv \mathbf{V}_{E \times B}, \quad \text { for the ion fluid, } \\
& \mathbf{u}_{e}=\mathbf{V}_{E \times B}+\frac{\nabla p_{e} \times \mathbf{B}}{e n_{e} B^{2}}, \text { for the electron fluid. }
\end{aligned}
$$

While the net current that flows by $\mathbf{E} \times \mathbf{B}$ drift is zero, the $\mathbf{E} \times \mathbf{B}$ flux value for the electrons is of interest since it gives the ion flux represented in terms of current since charge neutrality holds at the quite high level and ion diamagnetic drift is negligible. The vertical component of electron $\mathbf{E} \times \mathbf{B}$ drift current is given by

$$
I_{(\mathbf{E} \times \mathbf{B}) z}=2 \pi e \int n_{e} \frac{\left(\mathbf{E} \times B_{\phi} \widehat{\phi}\right) \cdot \widehat{Z}}{B^{2}} R d R .
$$

As shown in figure 16 this flux is nearly zero near the bottom electrode. It increases as $\mathrm{Z}$ increases over the mid-plane at $\mathrm{Z}=0$. In the case of $P_{i n j}=1 \mathrm{~kW}$, for example, the flux finally reaches $4 \mathrm{~A}$ for protons ( $-4 \mathrm{~A}$ for electrons) near the top electrode. The results including the diamagnetic drift current originated from the electron VTF-drift in addition to the $\mathbf{E} \times \mathbf{B}$ drift current indicates that the electron charge flux that flows into the top electrode is $-2 \mathrm{~A}$ and that of ions is $4 \mathrm{~A}$. Since the top panel collects the ion flux of $2 \mathrm{~A}$ and it is impossible for the electrons to reach the top panel across the pure 
toroidal field over the long distance of $6 \mathrm{~cm}$ of the limiter shadow, this result suggests that the top limiter collects simultaneously the ion current of $2 \mathrm{~A}$ and the electron current of $-2 \mathrm{~A}$, thus the limiter net current is zero as observed in figure $3(\mathrm{a})$. It does not necessarily mean no particle flux when the limiter current at the ion VTF drift side is zero. It means that the ion current and the electron current are balanced each other as the case occurred for a Langmuir probe at the floating potential. Actually when the probe tip is located in the shadow of the limiter at the ion VFT drift side its floating potential is nearly zero when $B_{Z}=0$. This result may have to do with that the field line is purely toroidal and the shadow region is not linked with the main room along the field line. When a finite $B_{Z}$ is applied both regions are connected by the helical field lines and the electron current flows into the limiter at the ion VFT drift side as shown in figures $3(\mathrm{~b})$ and 6 .

In every case presented in figure 16 the up-ward ion $\mathbf{E} \times \mathbf{B}$ current near the ion VTF drift side electrode is in excess of the electron VTF-drift current, which is needed for that all the electrons that circulate to the top electrode from the bottom electrode via the external circuit are recombined with the ions. In this region the potential descends towards the top electrode and, therefore the ion upward VTF drift raises the kinetic energy and then increases the VTF drift velocity, resulting in a positive feedback that may drive some ions to the top electrode as proposed theoretically in the next section 4.5. While there arises a positive feedback also for the electrons as well, the direction is downwards.

\subsection{Ion VTF drift enhanced by electrostatic field}

First, we investigate the electron and ion trajectories in a model space potential by solving the equation of motion with appropriate initial conditions. Figure 17 elucidates characteristics of ion orbits near the top electrode where the electrostatic potential significantly descends towards the top electrode and the ions drift upwards towards the electrode by the VTF drift as well as drift inwards towards the centre post by the $E_{Z} \widehat{Z} \times B_{\phi} \widehat{\phi}$ drift. The ions gain kinetic energy and their VTF drift velocity increases as they descends the potential slope, and finally some protons reach the top panel, handing off their charges to the panel to close the external return circuit. On the other hand, the ions which have large mass numbers can obtain a large Lamor radius towards the top electrode by descending the potential slope and easily reaches the electrode. This may be related with the relatively lower potential hill observed for the cases of $\mathrm{Ne}$ and Ar as shown in figure 13 .

Figure 18 shows various electron orbits in the potential hill near the top ion VFT drift side electrode. In the case with no ECR heating (ECH) low energy electrons do not reach the bottom electrode since their kinetic energy decreases as they descend the potential slope by the VTF drift toward the bottom electrode. With ECH some electrons can gain sufficient energy to reach the bottom electrode. Note that the trajectories of these heated electrons towards the bottom electrode are coincide with the tilted density 

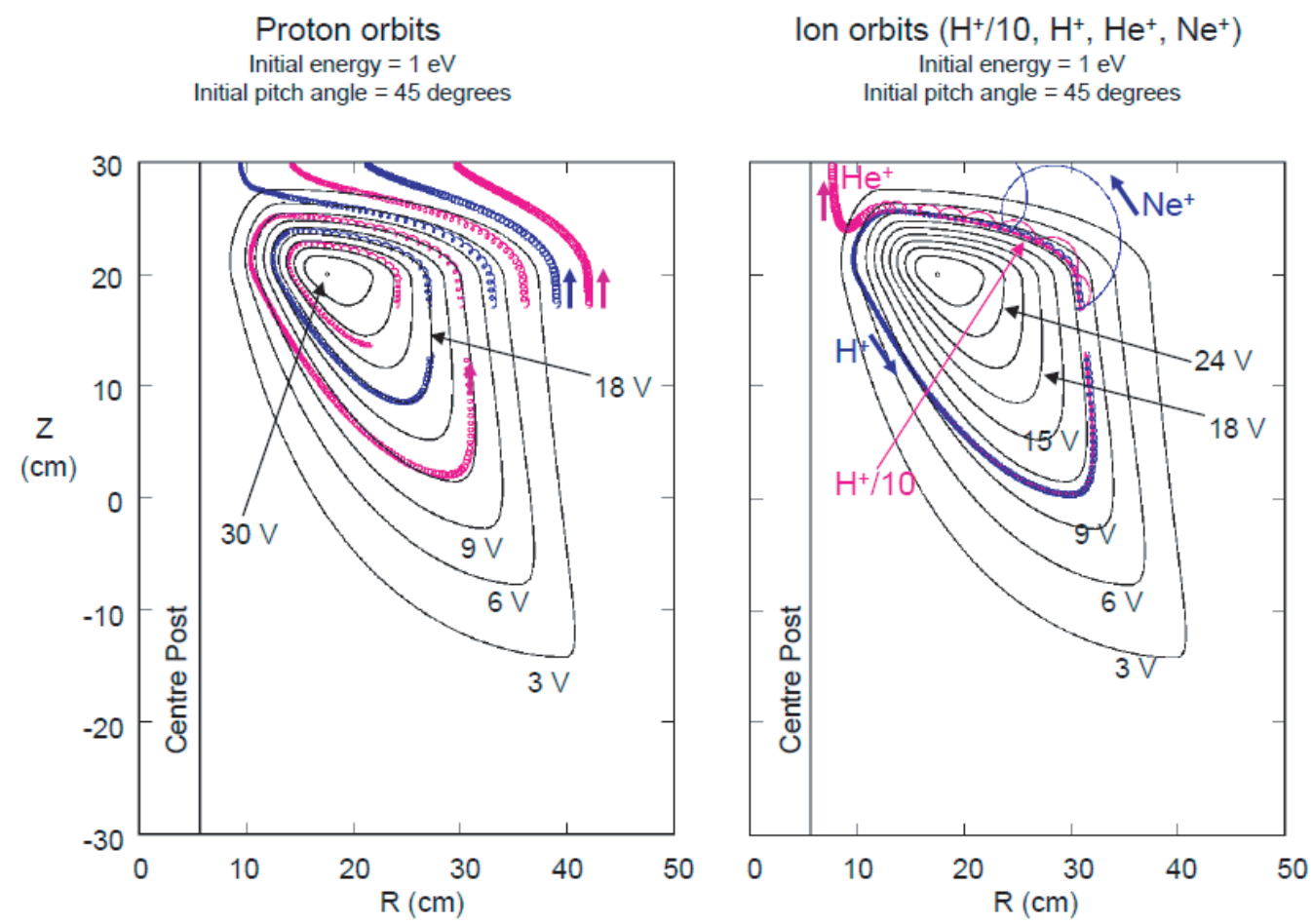

Figure 17. Various ion orbits in the potential hill near the top ion VFT drift side electrode. $H^{+} / 10$ means the one tenth of proton mass.

ridge for the cases of $B_{Z}=0$ as seen in figures $9-11$.

An analytical formula that elucidates this drift of protons may be obtained for a simplified electrostatic potential. We assume an electrostatic potential $V_{S}$ that depends only on Z, $V_{S}=V_{S}(Z)$, and monotonically descends towards the top panel at $Z=Z_{T P}$, where $V_{S}\left(Z_{T P}\right)=0$. Suppose a proton that locates initially at $R=R_{0}$ and $Z=Z_{0}$ and has an initial velocity $v_{0}$ and an initial pitch angle $\alpha_{0}$. We write that $V_{S}\left(Z_{0}\right)=V_{S 0}$. The protons drift inwardly as well as upwardly as described above, making a drift orbit on the poloidal cross section. Along this orbit there are three constants of motion; total energy $U$, angular momentum $L$ and magnetic moment $\mu$ as follows.

$$
U=\frac{M}{2}\left(v_{\|}^{2}+v_{\perp}^{2}\right)+q V_{S}, \quad L=M R v_{\|}+q R A_{\phi}, \quad \mu=\frac{M v_{\perp}^{2}}{2 B_{\phi}} .
$$

We analyze the case of no vertical magnetic field for simplicity, that is, $A_{\phi} \equiv R B_{Z} / 2=0$ Then,

$$
U=\frac{L^{2}}{2 M R^{2}}+\frac{\mu B_{0} R_{0}}{R}+q V_{S}(Z)=\frac{L^{2}}{2 M R_{0}^{2}}+\frac{\mu B_{0} R_{0}}{R_{0}}+q V_{S 0}
$$

From above relation we obtain,

$$
\frac{R_{0}}{R}=-\frac{1}{2} \tan ^{2} \alpha_{0}+\sqrt{\left(\frac{1}{2} \tan ^{2} \alpha_{0}+1\right)^{2}+\frac{q\left(V_{S 0}-V_{S}\right)}{W_{0}} \frac{1}{\cos ^{2} \alpha_{0}}},
$$



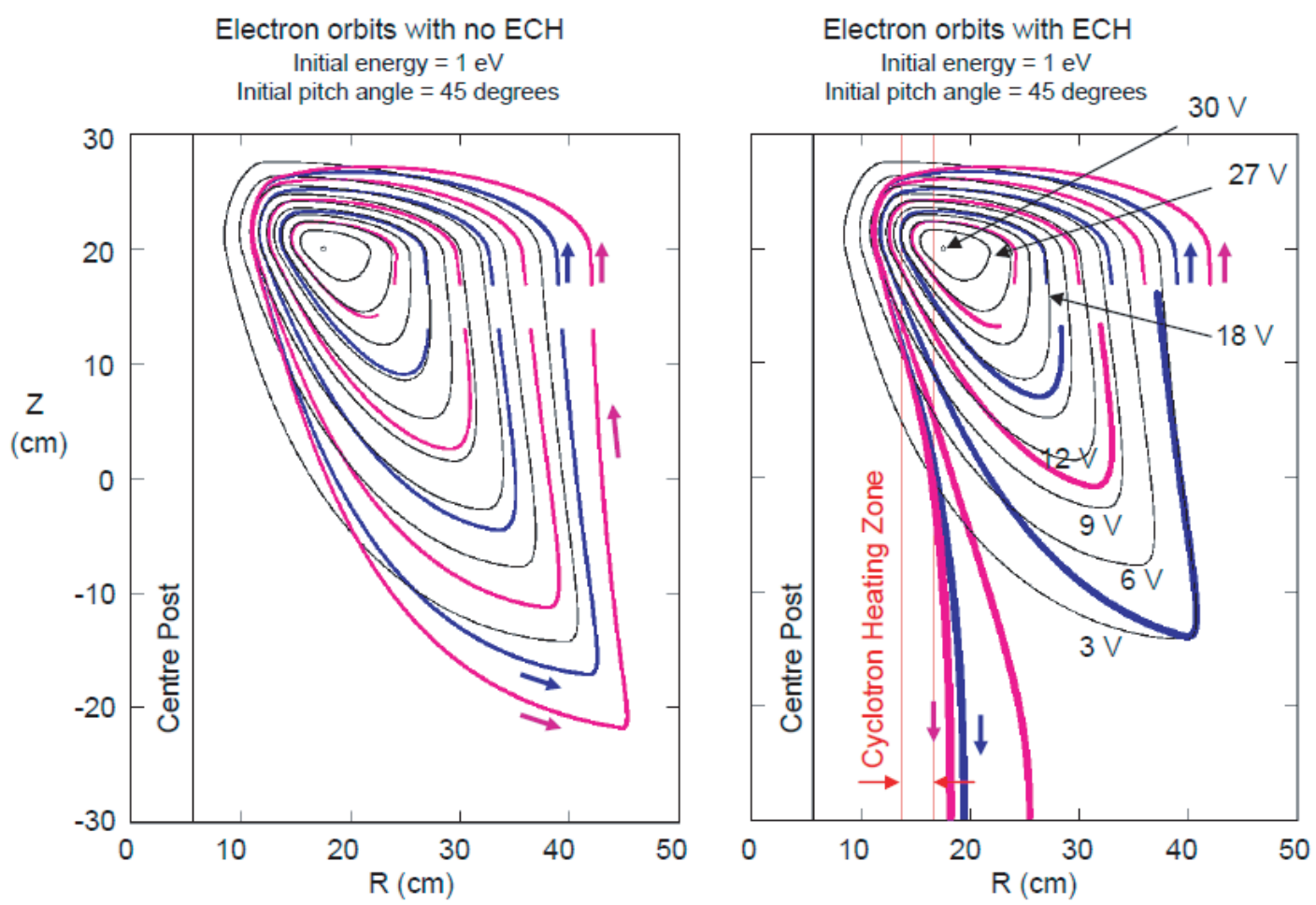

Figure 18. Various electron orbits in the potential hill near the top ion VFT drift side electrode. In the case of no ECH the electrons do not reach the bottom electron VTF drift side electrode. With ECH some electrons can reach the bottom electrode. In order to see the ECH effect by the change of Larmor radius the electron mass assumes one tenth of proton mass in the calculation.

where $W_{0}=M v_{0}^{2} / 2$ is the initial kinetic energy. The radial position on the top panel on which the proton arrives, $R_{T P}$ is given by setting $V_{S}=0$ in the above equation. We consider the case of $q V_{S 0}>>W_{0}$. Especially, when $\left|\alpha_{0}\right|<<1$,

$$
\frac{R_{T P}}{R_{0}} \simeq \sqrt{\frac{W_{0}}{q V_{S 0}+W_{0}}}\left(1-\frac{1}{2} \sin ^{2} \alpha_{0}\right) \approx \sqrt{\frac{W_{0}}{q V_{S 0}}} .
$$

When $\left|\alpha_{0}\right| \approx \pi / 2$,

$$
\frac{R_{T P}}{R_{0}} \simeq \frac{W_{0} \sin ^{2} \alpha_{0}}{W_{0} \sin ^{2} \alpha_{0}+q V_{S 0}\left(1-\frac{q V_{S 0}}{W_{0}} \frac{1}{\tan ^{2} \alpha_{0} \sin ^{2} \alpha_{0}}\right)} \approx \frac{W_{0}}{q V_{S 0}} .
$$

In conclusion, the radial location on which protons reach is compressed from the initial location as

$$
\frac{R_{T P}}{R_{0}} \approx \frac{W_{0}}{q V_{S 0}} \sim \sqrt{\frac{W_{0}}{q V_{S 0}}} \quad \text { for } \quad \alpha_{0}=\frac{\pi}{2} \sim 0
$$

Thus, the compression rate depends on the initial pitch angle and the ratio of initial kinetic energy to the initial potential energy. It is straightforward to show that the relationship given by equation (20) holds also when there is a finite $B_{Z}$ in the order of the present experiments $\left(\left|B_{Z}\right| \leq 6 \mathrm{G}\right)$. 
Even if above model of potential-slope-enhanced-ion-VTF-drift does work, it is remarkable that the charge neutrality is kept at the very high level under the constraint for the pressure profile in the plasma, that is, the pressure profile is uniform along the vertical direction due to the constraint of $\nabla \cdot \mathbf{j}=0$ under $B_{Z}=0$. The $\mathbf{E} \times \mathbf{B}$ circulation of charged particles may play a crucial role to mix up the charged particles to keep charge neutrality. The average lifetime of charged particles after the birth by ionization until the recombination at the wall surface may be estimated as $\tau=e N_{e} / I_{e}$, where $I_{e}$ is the electron current flowing into the electrodes and $N_{e}$ is the number of electrons in the vessel. The result is $\tau=0.3 \sim 0.5 \mathrm{~ms}$. The charged particles cover only $0.6 \sim 1 \mathrm{~m}$ by the $\mathbf{E} \times \mathbf{B}$ drift velocity of $2000 \mathrm{~ms}^{-1}$ before recombination. This distance corresponds to only one cycle of rotation around the potential peak, suggesting that one cycle of rotation is sufficient to keep charge neutrality, again being quite remarkable.

\subsection{Equilibrium and current circulation under weak $B_{Z}$}

4.6.1. Force balance along the field line There arises a toroidal plasma current when a finite $B_{Z}$ is applied, suggesting an electron flow along the helical field lines. The simplified fluid equation for the electron force balance parallel to the magnetic field is deduced from equation (6) as follows,

$$
0=-e n_{e} E_{\|}-\frac{\partial p_{e}}{\partial \ell}+e n_{e} \eta j_{\|}
$$

where $e n_{e} \eta j_{\|}=R_{e i \|}$ describes the collisional momentum transfer between electrons and ions, $\ell$ is the length along the field line and $\eta[\Omega \cdot \mathrm{m}]=7.6 \times 10^{-4} T_{e}^{3 / 2}\left(T_{e}\right.$ is in eV) is the Spitzer resistivity for the range of present experiment of $T_{e}=2 \sim 14 \mathrm{eV}$ and $n_{e}=(1 \sim 10) \times 10^{10} \mathrm{~cm}^{-3}$. Equation $(21)$ represents Ohm's law when the first and third terms are leading terms and the second term is negligible. In this case the toroidal current density is given by

$$
j_{\phi}=\frac{E_{\|}}{\eta}
$$

Figure 19 shows a comparison of predictions for the toroidal current from the Ohm's law model with the magnetic measurement. The magnetic results are obtained by analyzing the data from the flux loops shown in figure 2 [23]. The power supplies for the poloidal field coils and the toroidal field coil are all transistor-regulated without thyristor switching circuit. Furthermore, all the coil currents are steady in the present experiments. These conditions realize low noise measurement and the results are reliable. The figure shows that not only the current magnitude, but the current location are quite different from the Ohm's law prediction. This result indicates that the Ohm's law is not appropriate and the third term is negligible compared with the first and second terms in equation (21). Then the parallel force balance equation is approximately given by

$$
\frac{\partial p_{e}}{\partial \ell}=-e n_{e} E_{\|} \text {. }
$$



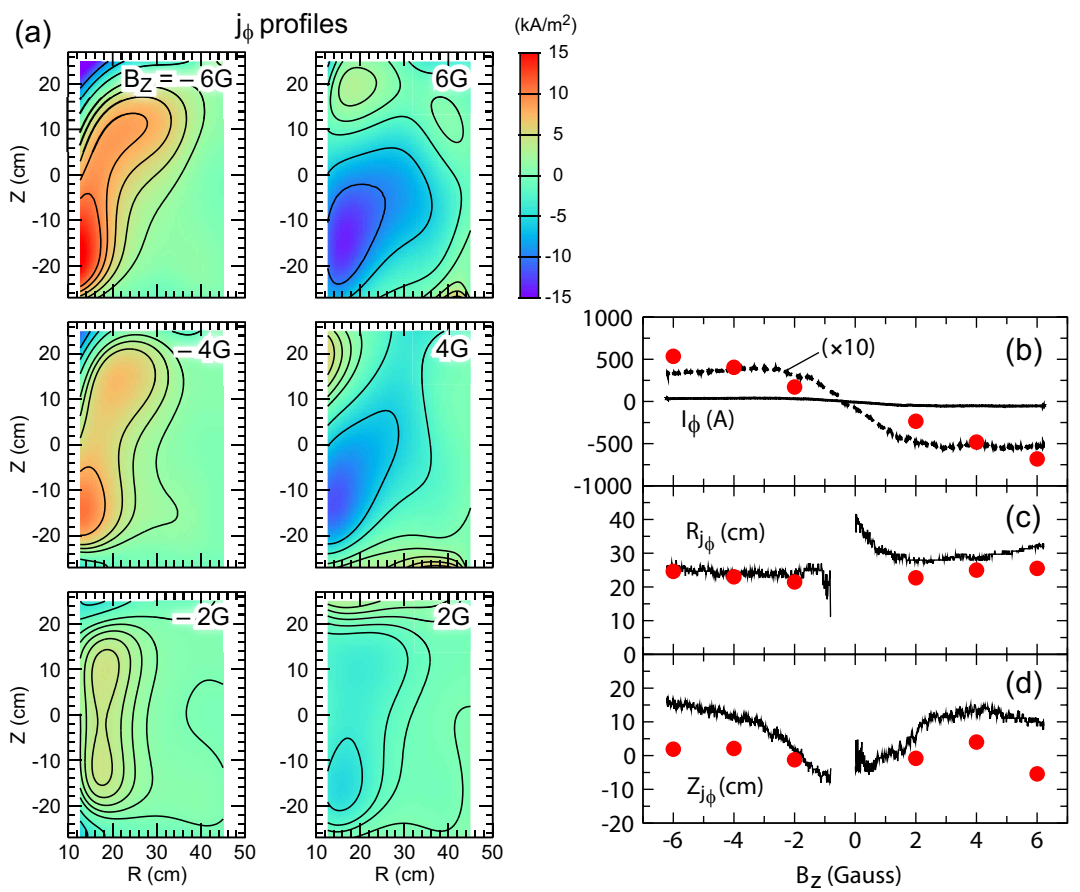

Figure 19. (a): Predictions of the toroidal current profiles by the Ohm's law model with the Spitzer resistivity. Some smoothing has been done on the $V_{S}$ profiles in figure 10 for present numerical analysis. (b), (c) and (d): Comparisons of the Ohm's law predictions (red solid circles) with the magnetic results for the current magnitude, radial and vertical locations of current centre, respectively.

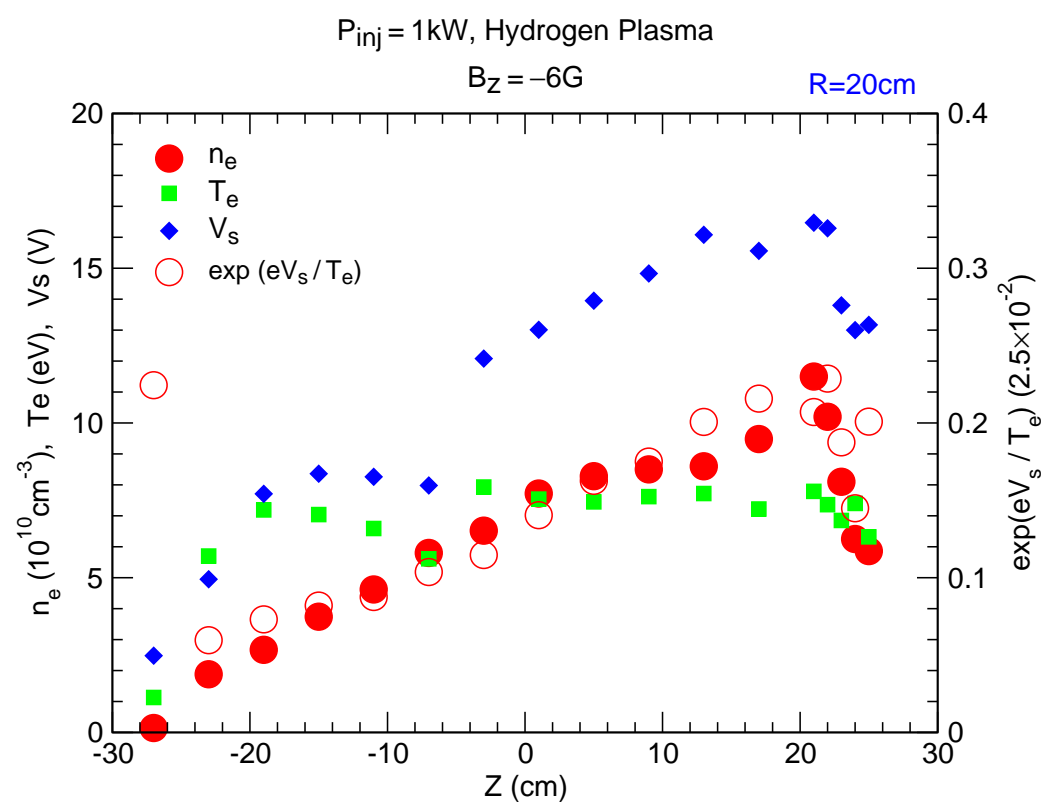

Figure 20. Equilibrium profiles of the electron density and temperature and space potential along a helical field line. 
Figure 20 shows that the electrons are isothermal along the helical field line. Then equation (23) predicts that the electron density obeys the Boltzmann law for the space potential as

$$
n_{e} \propto \exp \left(e V_{S} / T_{e}\right)
$$

so as to satisfy the parallel force balance of electron fluid along the field lines. This is the case as seen in figure 20 .

4.6.2. Toroidal current for radial force balance Equation (14), which is the solution for the perpendicular force balance under the constraint of divergence free of the current, gives the following formula for the toroidal current density.

$$
j_{\phi}(R, Z)=-\frac{2\left[p_{e}(R, Z)-p_{0}(R)\right]}{R B_{Z}}
$$

This has the same form as equation (3) which represents the toroidal current density appeared as a return current along the helical field lines in the particle drift picture. This suggests that the base portion $p_{0}$ may correspond to the current that circulates via the external circuit and the excess portion $\left(p_{e}-p_{0}\right)$ may correspond to the internal return current that appears as the toroidal current. Actually, the central electron pressure is higher than the edge pressure near the electrodes at the same radial coordinate $\mathrm{R}$ as seen in the cases of $B_{Z}=-6$ and $6 \mathrm{G}$ in figures $9-12$. This result indicates that the vertical charge separation current density associated with the VTF-drift is also higher in the higher pressure region in the main room compared with the area near the electrodes. The excess portion of this VTF vertical current associated with the excess portion of pressure $\left(p_{e}-p_{0}\right)$ must flow down along the helical field lines so as to fulfill the constraint $\nabla \cdot \mathbf{j}=0$, resulting in the generation of a toroidal current.

We look for the reference horizontal plane of $Z=Z_{\text {ref }}$, on which $p_{0} \equiv p_{e}\left(R, Z_{\text {ref }}\right)$ and the electron VTF drift current given by equation (16) is equal, as a zeroth order approximation, to the current flowing out from the bottom electrode as shown in figure 21(b) by the following equation,

$$
I_{B L}=I_{Z}^{e}\left(Z=Z_{r e f}\right) \equiv \frac{4 \pi}{R B_{\phi}} \int R p_{e}\left(R, Z_{r e f}\right) d R .
$$

Thus

$$
j_{\phi}(R, Z)=-\frac{2\left[p_{e}(R, Z)-p_{e}\left(R, Z_{r e f}\right)\right]}{R B_{Z}}
$$

The toroidal current distributions given by equation (27) at various $B_{Z}$ values are shown in figure 21(a). The total currents and locations of current centre at various $B_{Z}$ values are compared with those measured magnetically in figures $21(\mathrm{c})-(\mathrm{e})$, respectively. While consistency of the prediction with the experimental results is improved drastically compared with the case of Ohm's law prediction, there still remains significant difference in the prediction of the toroidal current and the vertical location of the current centre.

Next, we examine the case with another reference plane of $Z=Z_{r e f}$, with which equation (27) gives the same toroidal current as that magnetically estimated from the 

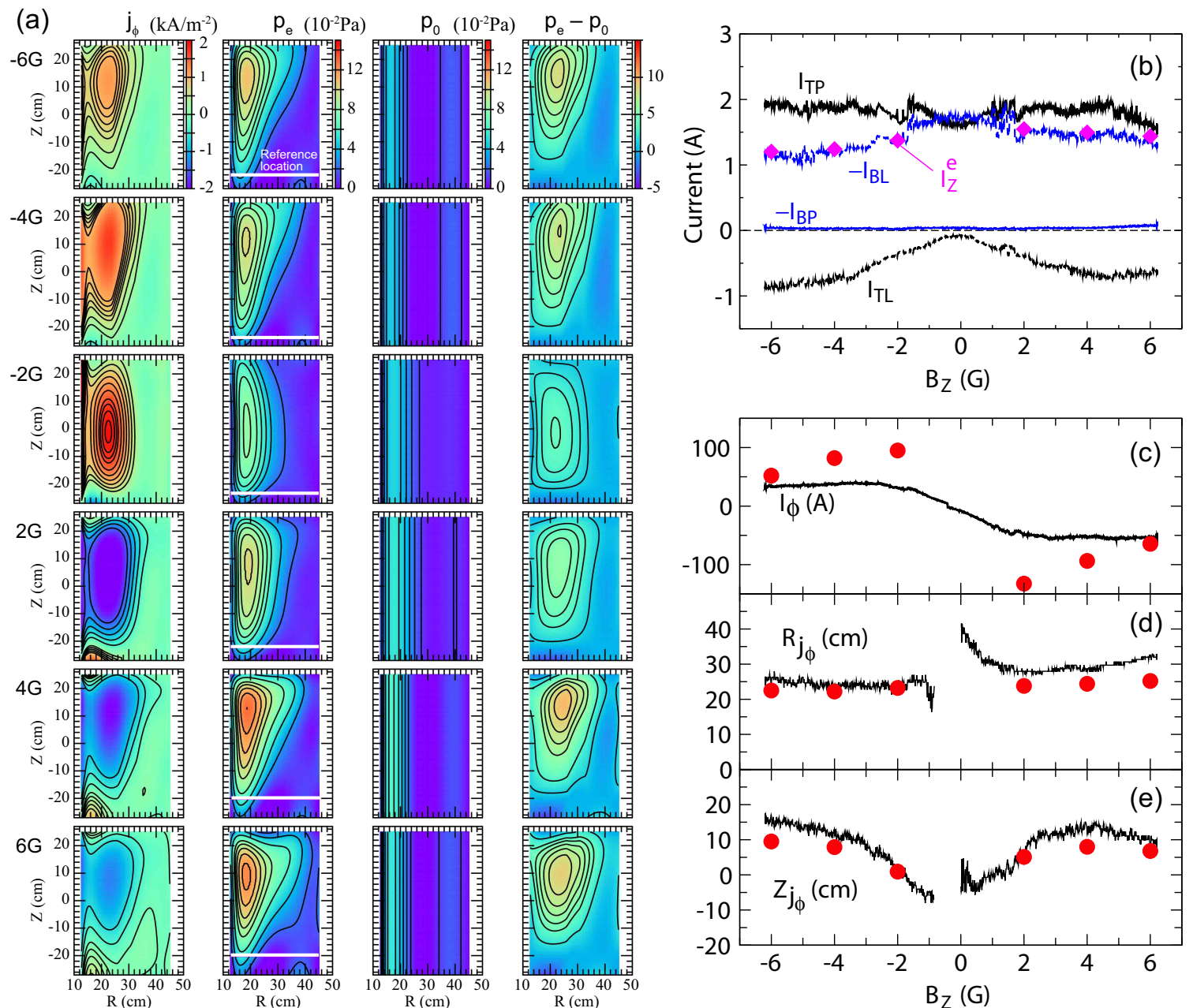

Figure 21. (a): Equilibrium toroidal current profiles based on the electron pressure profile with the reference location given by equation (26). Some smoothing has been done on the $p_{e}$ profiles in figure 10 for present numerical analysis. (b): Various electrodes currents. (c), (d) and (e): Comparisons of the equilibrium predictions (red solid circles) with the magnetic results for the current magnitude, radial and vertical locations of current centre, respectively.

flux loop signals.

$$
I_{\phi}(\text { magnetic result })=\int j_{\phi} d S
$$

The current distributions given by equations (27) and (28) at various $B_{Z}$ values are shown in figure $22(\mathrm{a})$. The locations of current centre at various $B_{Z}$ values are compared with those estimated magnetically from the flux loop signals in figures $22(\mathrm{~d})$ and (e), showing a better coincidence compared with the previous case in figures 21(d) and (e). The external circulation current predicted by equation (16) by using the pressure profile at this reference plane is roughly coincident with the current that flows out from the bottom electrode, although some difference still remains between them. Above results suggest that the observed currents are, in a zeroth order approximation, equilibrium 
(a)
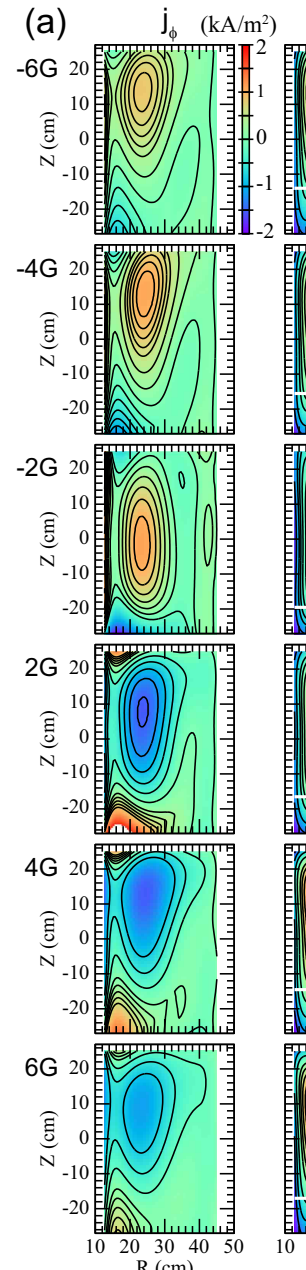

$\mathrm{R}(\mathrm{cm})$ $\mathrm{p}_{\mathrm{e}}\left(10^{-2} \mathrm{~Pa}\right)$
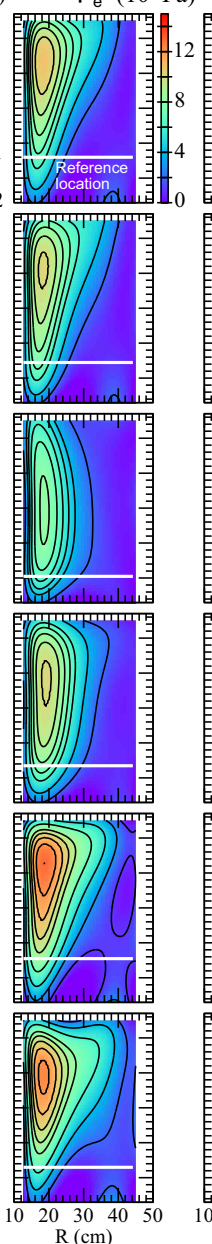

$\mathrm{p}_{0}\left(1^{-2} \mathrm{~Pa}\right)$
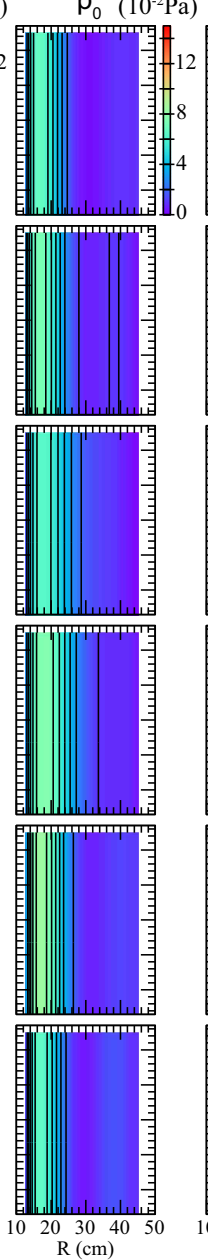

$p_{e}-p_{0}$

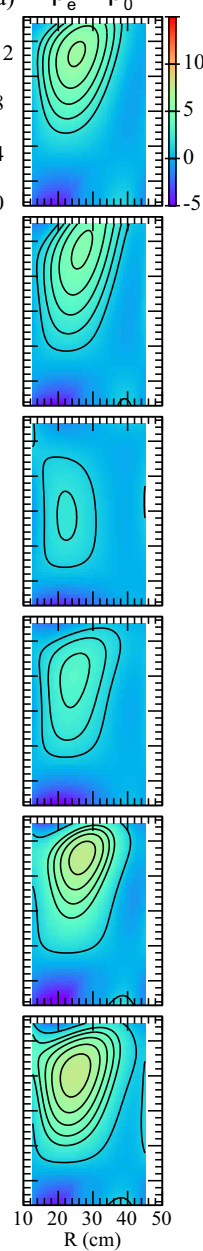

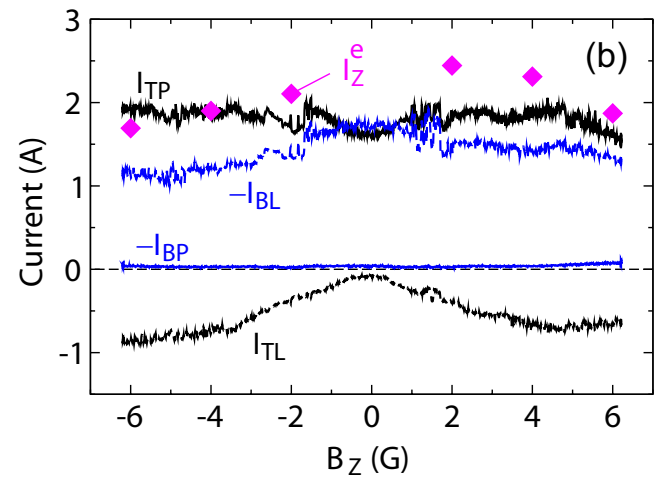

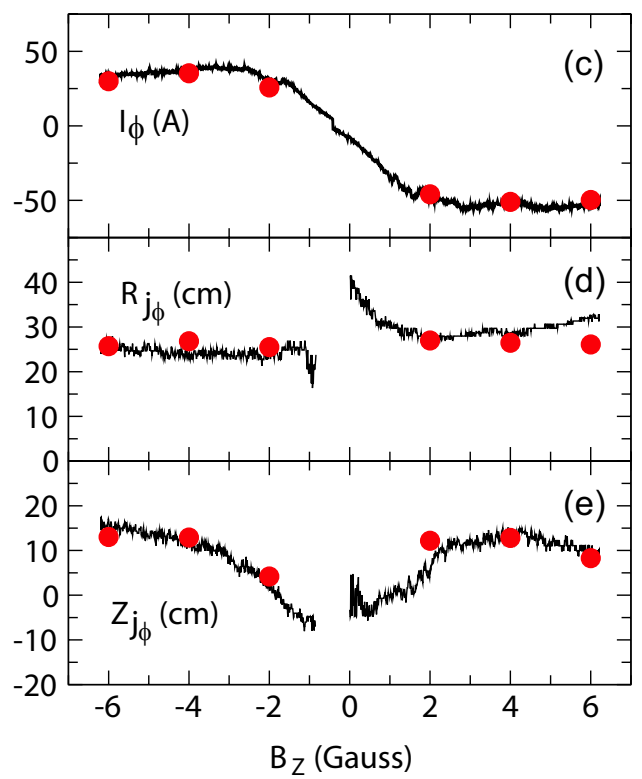

Figure 22. (a): Equilibrium toroidal current profiles based on the electron pressure profile with the reference location given by equation (28). Some smoothing has been done on the $p_{e}$ profiles in figure 10 for present numerical analysis. (b): Various electrodes currents. In this reference case adjusted for the toroidal current the diamagnetic current $I_{Z}^{e}$ somewhat differs from the bottom limiter current $I_{B L}$. (c), (d) and (e): Comparisons of the equilibrium predictions (red solid circles) with the magnetic results for the current magnitude, radial and vertical locations of current centre, respectively.

currents for the radial force balance under the external toroidal and vertical fields. The ratio of the counterbalancing force from the toroidal current to that from the vertical current is estimated by equation (5) and found to increase with $B_{Z}$ and becomes more than unity when $\left|B_{Z}\right|=6 \mathrm{G}$ in figure 22 . While this return current model for circulation of the vertical charge separation current is phenomenological one, overall consistency with the experiments suggests that the model essentially reflects the physics responsible for the toroidal current generation under a weak $B_{Z}$ in the present toroidal ECR plasmas.

The vertical components of the self fields by the toroidal currents of $\sim 50 \mathrm{~A}$ in figure 22 (c) is lower than $1 \mathrm{G}$, which is much smaller than the external vertical field of 
$B_{Z}=2 \sim 6 \mathrm{G}$, justifying the application of the equilibrium toroidal current model under the external field $B_{Z}$ given by equation (25). The previous result [17] shows that during a microwave pulse with a much larger power of $P_{i n j} \geq 5 \mathrm{~kW}$ and a stronger vertical field of $B_{Z} \geq 10 \mathrm{G}$ than the present experiments, the toroidal current temporally increases further, resulting in the formation of a closed field structure. While the equilibrium toroidal current given by equation (25) can not close the filed lines, it modifies the poloidal field from the initial external $B_{Z}$ field and induces secondary mechanisms of toroidal current generation that close the field lines as discussed in [15, 17]. After the formation of a closed flux surface the electron cyclotron current drive works to maintain and ramp up the toroidal current [17, 24].

Again concerning the present study, the remained question is how is determined the radial distribution of the electrode current at the top and bottom. The distribution is in the zeroth order approximation given by the reference pressure profile determined by equations (27) and (28) as shown in figure 22(a), where the reference pressure rapidly increases near the ECR layer towards the centre post. In addition to the ECR heating of electrons to drive electrons downward by the VTF drift as shown in figure 18, the characteristics of ion orbit near the top panel described in the previous section 4.5 and in figure 17, that is, the compression of ion radial coordinate during the VTF drift towards the top panel may be a candidate for this distribution.

\section{Summary}

In axisymmetric toroidal plasmas immersed in a toroidal field of $B_{\phi}$ in the cylindrical coordinates of $\mathrm{R}, \varphi$ and Z, the electrons and ions VTF-drift oppositely along the vertical direction due to the field gradient and curvature, driving the vertical charge separation current. The electrons that reach the bottom would flow through the conducting vessel to the top where they recombine with the ions, completing the current circulation. Helical field lines by the superposition of a weak vertical field $B_{Z}$ would provide another return path for the electrons, in which case the electrons circulate toroidally without vertical net drift, generating a toroidal current. These conjectures have been examined to be essentially correct on ECR-heated plasmas in the LATE device.

In the first series of experiments, the external circuit is artificially blocked during discharges. The discharges terminate upon the blocking when $B_{Z}=0$, while they survive with a toroidal current when $B_{Z}$ is large enough to provide internal return path in the plasma. This shows most directly that current circulation is vital to maintain the discharge.

In the second and third series of experiments, two dimensional profiles of the electron temperature, density and space potential are measured, by scanning a motordrive Langmuir probe by shot by shot basis, for hydrogen and noble gas plasmas under various $B_{Z}$ field. These various plasma profiles as well as the vertical and toroidal currents in the plasma obey the mirror symmetry upon the change of directions of the poloidal coil currents as well as the toroidal coil current, indicating that the 
measured profiles are reliable and essentially reflect characteristics of the equilibria under circulation of the vertical charge separation current. The characteristics are quite different with and without $B_{Z}$.

When $B_{Z}=0$, the electron pressure profile is approximately a uniform vertical ridge just outside the ECR layer, showing the strong constraint of $\nabla \cdot \mathbf{j}=0$ for the electron vertical charge separation current for this special case of no return path in the plasma. The space potential is broad with a significant positive peak near the top electrode. The analyses show that this nested potential drives the ions to the vicinity of the top electrode via $\mathbf{E} \times \mathbf{B}$ drift along the equipotential contours, where some ions may VTFdrift down the potential slope by a positive feedback and reach the top electrode. The space potential decreases with the mass of ions, suggesting importance of ion dynamics near the top electrode of the ion VTF-drift side.

When a weak $B_{Z}$ is applied, a toroidal current appears in addition to the vertical current. As $B_{Z}$ increases both the electron density and pressure profiles shift to the ion VTF drift direction, and also expands towards the weak field side, showing relaxation of the constraint under $B_{Z}=0$. The space potential $V_{S}$ is also relaxed and the electron density approximately obeys the Boltzmann law as $n_{e} \propto \exp \left(e V_{S} / T_{e}\right)$. The vertical and toroidal currents are found to be equilibrium currents driven by the base and excess portions of electron pressure, respectively, to ensure the radial force balance of the plasma torus. Contribution of toroidal current in this force force increases with $B_{Z}$.

\section{Acknowledgment}

The present work was supported by the KAKENHI and NIFS collaboration program in Japan.

\section{References}

[1] Peng Y-K M, Borowski S K and Kammash T 1978 Nucl. Fusion 181489

[2] Nakao S, Ogura K, Terumichi Y and Tanaka S 1983 Phys. Lett. A 96405

[3] Gilgenbach R M et al 1981 Nucl. Fusion 21319

[4] Gribov Y, Humphreys D, Kajiwara K, Lazarus E A, Lister J B, Ozeki T, Portone A, Shimada M, Sips A C C and Wesley J C 2007 Nucl. Fusion 47 S385

[5] Kubo S, Nakamura M, Cho T, Nakao S, Shimozuma T, Ando A, Ogura K, Maekawa T, Terumichi Y and Tanaka S 1983 Phys. Rev. Lett. 501994

[6] Toi K et al 1984 Phys. Rev. Lett. 522144

[7] Parail V V, Pereverzev G V and Vojtsekhovich I A 1985 Proc. 10th Int. Conf. on Plasma Physics and Controlled Nuclear Fusion (London, 1984) vol 1, p 605, IAEA-CN-44/F-IV-4 (Vienna: IAEA)

[8] Zakharov L E and Pereverzev G V 1988 Sov. J. Plasma Phys. 1475

[9] Müller S H, Fasoli A, Labit B, McGrath M, Podestá M, and Poli F M 2004 Phys. Rev. Lett. 93 165003

[10] Podestá M, Fasoli A, Labit B, McGrath M, Müller S H and Poli FM 2006 Plasma Phys. Control. Fusion 481053

[11] Furno I et al 2008 Phys. Rev. Lett. 100055004 
[12] Perez J C, Horton W, Gentle K, Rowan W L, Lee K and Dahlburg R B 2006 Phys. Plasmas 13 032101

[13] Rypdal K, Grønvoll E, Ømynes F, Fredriksen Å, Armstrong R J, Trulsen J and Pétcseli H L 1994 Plasma Phys. Control. Fusion 361099

[14] Forest C B, Hwang Y S, Ono M, and Darrow D 1992 Phys. Rev. Lett. 68, 3559

[15] Forest C B et al. 1994 Phys. Plasmas 11568

[16] Maekawa T et al 2005 Nucl. Fusion 451439

[17] Yoshinaga T, Uchida M, Tanaka H, and Maekawa T 2006 Phys. Rev. Letters 96125005.

[18] Yoshikawa S, Harries W I and Sinclair R M 1963 Phys. Fluids 61506

[19] Nishi S, Sakabe T, Uchida M, Tanaka H and Maekawa T, 2010 Plasma Phys. Control. Fusion 52 065011

[20] Geller R, Electron Cyclotron Resonance Ion Sources and ECR plasma, (Institute of Physics Publishing, Bristol and Philadelphia, 1996)( see section 4.1)

[21] Goldston R J and Rutherford P H, Introduction to Plasma Physics, (Institute of Physics Publishing, Bristol and Philadelphia, 1995)( see Section 8.1)

[22] ibid. (see Section 7.2 and Figure 7.2)

[23] Yoshinaga T, Uchida M, Tanaka H, Maekawa T, 2007 Nucl. Fusion 47210.

[24] Uchida M, Yoshinaga T, Tanaka H, and Maekawa T 2010 Phys. Rev. Letters 104065001. 